\title{
Synthesis and Determination of Physicochemical Properties of New 3-(4-Arylpiperazin-1-yl)-2- hydroxypropyl 4-Alkoxyethoxybenzoates
}

\author{
Pavlina Marvanova ${ }^{1, *}$, Tereza Padrtova ${ }^{1}$, Klara Odehnalova ${ }^{1}$, Ondrej Hosik ${ }^{1}$, Michal Oravec ${ }^{2}$ \\ and Petr Mokry ${ }^{1, *}$ \\ 1 Department of Chemical Drugs, Faculty of Pharmacy, University of Veterinary and Pharmaceutical Sciences, \\ Palackeho 1, 61242 Brno, Czech Republic; padrtova.tereza@seznam.cz (T.P.); odehnalovak@vfu.cz (K.O.); \\ f12058@vfu.cz (O.H.) \\ 2 Global Change Research Institute CAS, Belidla 986/4a, 60300 Brno, Czech Republic; oravec.m@czechglobe.cz \\ * Correspondence: marvanovap@gmail.com (Pa.M.); mokryp@vfu.cz (Pe.M.); Tel.: +420-541-562-928 (Pe.M.)
}

Academic Editor: Atanas G. Atanasov

Received: 27 September 2016; Accepted: 30 November 2016; Published: 7 December 2016

\begin{abstract}
Nine new dihydrochloride salts of 3-(4-arylpiperazin-1-yl)-2-hydroxypropyl 4-alkoxyethoxybenzoates were designed and synthesized. The physicochemical properties such as lipophilicity index $\left(\log k_{w}\right)$ and dissociation constant $\left(\mathrm{p} K_{\mathrm{a}}\right)$ were experimentally determined and compared to the software calculated data. The lipophilicity index was determined by means of reversed-phase high performance liquid chromatography (RP-HPLC). The $\mathrm{p} K_{\mathrm{a}}$ values were determined by means of capillary zone electrophoresis. The "drug-likeness" properties according to the Lipinski Rule of Five and prediction of possible blood-brain barrier penetration were computed and discussed.
\end{abstract}

Keywords: arylcarbonyloxyaminopropanols; phenylpiperazines; $\mathrm{p} K_{\mathrm{a}}$ determination; lipophilicity index; Lipinski rules; blood-brain barrier

\section{Introduction}

Nowadays, many drugs used in clinical practice have acidic or basic functionalities in their structures, antagonists of $\beta$-adrenergic receptors ( $\beta$-blockers) included. $\beta$-blockers, one of the most prescribed and used cardiovascular drugs for more than 50 years, are still being improved with additional features such as vasodilatory and antioxidant activity. The dual $\alpha_{1}$ - and $\beta$-blockers (e.g., carvedilol, labetalol, vanylidilol) showed hemodynamic benefits, including reduction of peripheral vascular resistance, improvement of cardiac output and left ventricular function, which is why the vasodilatory activity is an enhancement to the antihypertensive effect of $\beta$-blockade and offsets its drawback [1-3]. The pharmacokinetic effect and toxicity of the newly prepared compound can be predicted also from the determination of their physicochemical properties $[4,5]$.

The physicochemical parameters such as lipophilicity $(\log P)$, distribution coefficient $(\log D)$ and dissociation constant $\left(\mathrm{p} K_{\mathrm{a}}\right)$ of an active pharmaceutical ingredient belong to crucial properties used to estimate the absorption, distribution, metabolism, and excretion (ADME) of a compound in biological systems as well as quantitative structure-activity and structure property relationship (QSAR/QSPR) profiling [6-8]. There are a number of commercially available programs that can predict these values, but the results of prediction can considerably differ depending on the algorithm used [9]. Therefore, predicted values should be replaced by experimental data in early stage of drug development. Among others, the reversed-phase high performance liquid chromatography (RP-HPLC) method is a successfully used tool for lipophilicity assessment [10-12]. One of the most predictive methods for lipophilicity determination is realized by monitoring the logarithmic retention factor 
$\left(\log k_{i}\right)$ of the analyte with changing ratio of organic mobile phase fraction. Isocratic retention factors are extrapolated to $100 \%$ water to obtain $\log k_{w}^{a p p}$ values at a given $\mathrm{pH}$. The dissociation constant $\mathrm{p} K_{\mathrm{a}}$ can be commonly determined by means of potentiometric titration, UV-VIS spectroscopy, capillary electrophoresis (CE), RP-HPLC or NMR spectroscopy [13,14]. The determination by means of CE proved to be a suitable technique [15-17], where the $\mathrm{p} K_{\mathrm{a}}$ values are determined from monitoring dependence of effective mobility of the analyte on $\mathrm{pH}$ of the background electrolyte (BGE) using non-linear regression analysis.

This contribution is focused on the synthesis and experimental determination of the physicochemical properties of nine new 3-(4-arylpiperazin-1-yl)-2-hydroxypropyl 4-alkoxyethoxybenzoates. The benzene ring (a part of 4-hydroxybenzoic acid) in an arylcarbonyloxyaminopropanol fragment is substituted in the C4 position with a propoxyethoxy, isopropoxyethoxy or allyloxyethoxy chain. The basic part of the molecule is formed with phenylpiperazine fragment that is either unsubstituted or substituted with a methoxy moiety in the $\mathrm{C}^{\prime}$ or $\mathrm{C}^{\prime}$ position. All the compounds were prepared as dihydrochloride salts to increase their solubility in water. The newly synthesized agents are expected to have cardiovascular activity due to the presence of particular structures of pharmacophores (arylcarbonyloxyaminopropanol moiety responsible for $\beta$-adrenolytic activity [18], phenylpiperazine fragment providing $\alpha_{1}$-adrenolytic activity [19]), nevertheless, the biological activities of the discussed compounds have not been determined so far.

\section{Results and Discussion}

\subsection{Chemistry}

The final products were prepared via multi-step synthesis as described in Scheme 1. Tosylate intermediates T1a-c and $\mathbf{T} \mathbf{2}$ were prepared according to the published procedure (Scheme 2), and the spectroscopic and physical data were in agreement with those reported in the literature [20-23]. The reaction temperature $\left(0^{\circ} \mathrm{C}\right)$ and time (two to three hours) was crucial to obtain pure products at good yields. Intermediates 1-3 were already described in the literature but were prepared by a different synthetic method, and their spectroscopic data were not published yet. Intermediates 4-6 were prepared from ethyl 4-hydroxybenzoate via reaction with the appropriate tosylate intermediates T1a-c. The reaction was carried out in acetone with potassium carbonate at $65{ }^{\circ} \mathrm{C}$ for $12 \mathrm{~h}$ and then stirred for at least $12 \mathrm{~h}$ at ambient temperature, changing the solvent (acetonitrile, dimethylformamide) did not improve the purity nor the yield of the desired products. The hydrolysis was then performed by using a $2 \mathrm{M}$ solution of sodium hydroxide and the desired acids 4-6 were obtained by displacing with $\mathrm{HCl}$, extracting to chloroform and evaporating. The final products, hydrochloride salts $\mathbf{7 a - c}, \mathbf{8 a}-\mathbf{c}, \mathbf{9 a}-\mathbf{c}$, were prepared according to the already published synthesis [24]. Briefly, the appropriate acids 1-3 were transformed into the potassium salt using potassium hydroxide in a mixture of methanol and propan-2-ol (ratio 1:3). Compounds 4-6, (oxiran-2-yl)methyl 4-(2-alkoxyethoxy)benzoates were obtained by reaction of intermediate $\mathbf{T} 2$ and potassium 4-(2-alkoxyethoxy)benzoate in dimethylformamide for $7 \mathrm{~h}$ at $70^{\circ} \mathrm{C}$. The oxirane ring was then opened by appropriate phenylpiperazine, the reaction was carried out in propan-2-ol for one hour at $80^{\circ} \mathrm{C}$ and for $72 \mathrm{~h}$ at ambient temperature. The obtained bases were finally converted to their dihydrochloride salts by extend of hydrochloric acid to enhance the solubility of the compounds in water. The protonation of the phenylpiperazine nitrogens was not experimentally determined for the discussed final compounds but the hypothesis is based on the measurement carried out for similar structures [24]. 

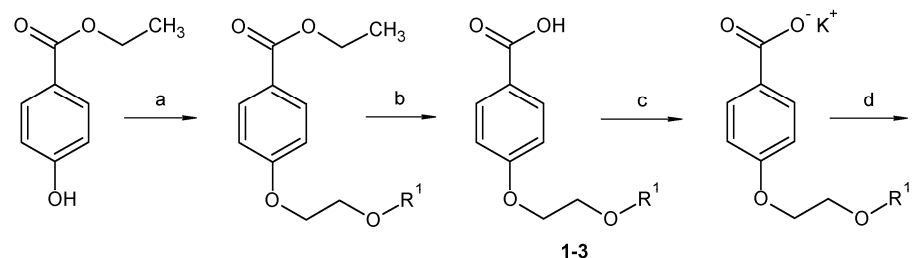

$1-3$
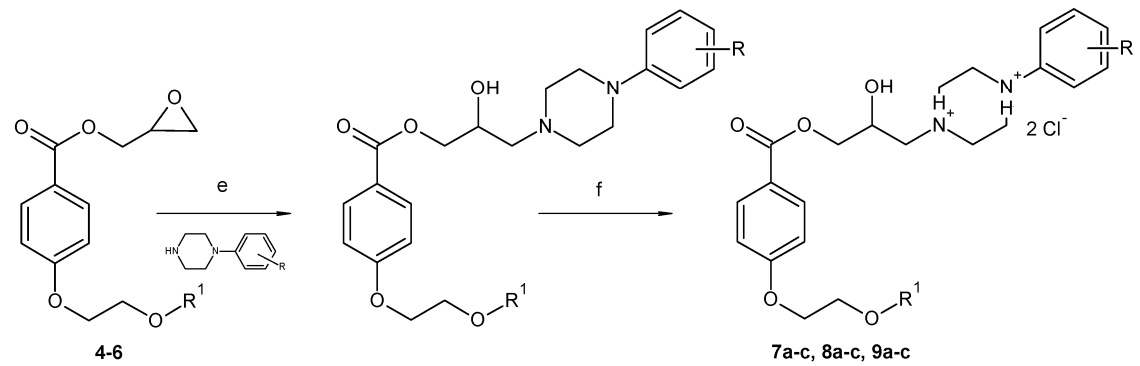

$R^{1}:$ (7) $\mathrm{CH}_{2}=\mathrm{CHCH}_{2} ;(8)\left(\mathrm{CH}_{3}\right)_{2} \mathrm{CH}$; (9) $\mathrm{CH}_{3} \mathrm{CH}_{2} \mathrm{CH}_{2}$

R: (a) $\mathrm{H}$; (b) $2-\mathrm{OCH}_{3}$; (c) $4-\mathrm{OCH}_{3}$

Scheme 1. Synthesis of discussed compounds 1-9a-c. Reagents and conditions: (a) $\mathbf{T} 1 \mathrm{a}-\mathbf{c}, \mathrm{K}_{2} \mathrm{CO}_{3}$, acetone, reflux; (b) $\mathrm{NaOH}, \mathrm{HCl}$, reflux; (c) $\mathrm{KOH}, \mathrm{MeOH}$, propan-2-ol (IPA); (d) T2; dimethylformamide (DMF), reflux; (e) arylpiperazine, IPA, reflux; (f) $\mathrm{HCl}, \mathrm{Et}_{2} \mathrm{O}$.

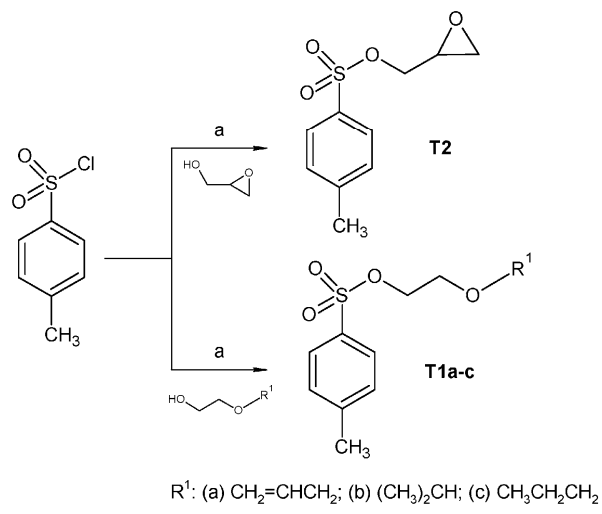

Scheme 2. Synthesis of intermediates T1a-c and T2. Reagents and conditions: (a) TEA, DCM, $0{ }^{\circ} \mathrm{C}$.

\subsection{Physicochemical Properties}

The dissociation constants $\left(\mathrm{p} K_{\mathrm{a}}\right)$ of studied compounds were determined by means of $\mathrm{CE}$. The measured values and values predicted by chemical software (ACD Percepta ver. 2012, Advanced Chemistry Development, Toronto, ON, Canada; MarvinSketch 6.0.6., ChemAxon Kft., Budapest, Hungary) are shown in Table 1 . The correlation coefficients of the experimentally determined values were greater than 0.999 and standard deviation equal or less than 0.02 units $(n=4)$. The experimental values are in the range 6.35-6.92 and the highest values were recorded for the 1-(2-methoxyphenylpiperazine) derivatives $\mathbf{7 b}, \mathbf{8 b}, \mathbf{9 b}$. The clinically used $\beta$-blockers are usually stronger bases [14], the values of studied compounds are lower due to the presence of piperazine moiety instead of secondary amine group.

The lipophilicity indexes $\log k_{w}^{7.4}$ were measured by means of RP-HPLC method. The $\log k_{w}$ values were then calculated according to Equation (2) given in Section 3.3. The correlation coefficients were greater than 0.999 with covariance equal or less than 0.03 units $(n=2)$. All the measured, calculated and predicted values are shown in Table 2.

The lipophilicity decreases in order from non-substituted through ortho- to para-substituted derivatives, and also in order from propoxy- through isopropoxy- to allyloxy-derivatives. 
Table 1. Experimentally determined $\mathrm{p} K_{\mathrm{a}}$ in comparison with calculated values of final compounds.

\begin{tabular}{|c|c|c|c|c|c|}
\hline Comp. & $\mathbf{R}^{1}$ & $\mathbf{R}$ & $p K_{\mathrm{a}}{ }^{\mathrm{a}}$ (exp.) & $\mathrm{p} K_{\mathrm{a}}{ }^{\mathrm{b}}$ (calc.) & $\mathrm{pK}_{\mathrm{a}}^{\mathrm{c}}$ (calc.) \\
\hline $7 a$ & allyl & $\mathrm{H}$ & $6.47 \pm 0.02$ & 6.60 & 7.70 \\
\hline $7 \mathrm{~b}$ & allyl & 2-MeO & $6.88 \pm 0.02$ & 6.70 & 7.40 \\
\hline $7 \mathrm{c}$ & allyl & 4-MeO & $6.49 \pm 0.02$ & 6.70 & 7.63 \\
\hline $8 a$ & isopropyl & $\mathrm{H}$ & $6.49 \pm 0.01$ & 6.60 & 7.70 \\
\hline $8 b$ & isopropyl & 2-MeO & $6.92 \pm 0.01$ & 6.70 & 7.40 \\
\hline $8 c$ & isopropyl & 4-MeO & $6.35 \pm 0.02$ & 6.70 & 7.63 \\
\hline $9 a$ & propyl & $\mathrm{H}$ & $6.50 \pm 0.02$ & 6.60 & 7.70 \\
\hline $9 b$ & propyl & 2-MeO & $6.79 \pm 0.02$ & 6.70 & 7.40 \\
\hline $9 \mathrm{c}$ & propyl & 4-MeO & $6.39 \pm 0.01$ & 6.70 & 7.63 \\
\hline
\end{tabular}

a Mean and standard deviation of four measurements; ${ }^{\mathrm{b}}$ calculated by ACD Percepta, ver. 2012; ${ }^{\mathrm{c}}$ calculated by MarvinSketch 6.0.6.

Table 2. Experimentally determined and calculated values of lipophilicity indexes $\left(\log k_{w}^{7.4}, \log k_{w}\right)$ in comparison with software-calculated values of distribution coefficients $\left(\log D_{7.4}\right)$ and lipophilicity $(\log P)$ of final compounds.

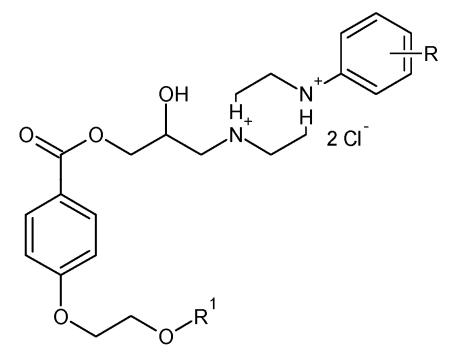

\begin{tabular}{|c|c|c|c|c|c|c|c|c|}
\hline Comp. & $\mathbf{R}^{1}$ & $\mathbf{R}$ & $\log k_{w}^{7.4 \mathrm{a}}$ & $\log D_{7.4}{ }^{b}$ & $\log D_{7.4}{ }^{\mathrm{c}}$ & $\log k_{w}$ & $\log P^{b}$ & $\log P^{c}$ \\
\hline $7 \mathbf{a}$ & allyl & $\mathrm{H}$ & $3.97 \pm 0.03$ & 3.97 & 3.15 & $4.01 \pm 0.09$ & 4.03 & 3.63 \\
\hline $7 \mathbf{b}$ & allyl & 2-MeO & $3.78 \pm 0.02$ & 3.47 & 3.17 & $3.89 \pm 0.08$ & 3.65 & 3.47 \\
\hline $7 c$ & allyl & 4-MeO & $3.67 \pm 0.02$ & 3.40 & 3.04 & $3.71 \pm 0.08$ & 3.55 & 3.47 \\
\hline $8 a$ & isopropyl & $\mathrm{H}$ & $4.21 \pm 0.01$ & 4.03 & 3.19 & $4.25 \pm 0.05$ & 4.09 & 3.67 \\
\hline $8 b$ & isopropyl & 2-MeO & $4.03 \pm 0.02$ & 3.38 & 3.21 & $4.15 \pm 0.07$ & 3.56 & 3.51 \\
\hline $8 c$ & isopropyl & 4-MeO & $3.90 \pm 0.02$ & 3.35 & 3.08 & $3.93 \pm 0.06$ & 3.50 & 3.51 \\
\hline $9 a$ & propyl & $\mathrm{H}$ & $4.29 \pm 0.03$ & 4.01 & 3.30 & $4.34 \pm 0.10$ & 4.07 & 3.78 \\
\hline $9 b$ & propyl & 2-MeO & $4.11 \pm 0.02$ & 3.49 & 3.32 & $4.20 \pm 0.09$ & 3.67 & 3.62 \\
\hline $9 c$ & propyl & 4-MeO & $3.99 \pm 0.02$ & 3.41 & 3.19 & $4.03 \pm 0.09$ & 3.56 & 3.62 \\
\hline
\end{tabular}

${ }^{a}$ Mean and standard deviation of four measurements; ${ }^{b}$ calculated by ACD Percepta, ver. 2012; ${ }^{c}$ calculated by MarvinSketch 6.0.6.

Moreover, other parameters, such as number of hydrogen bond donors (nOHNH), number of hydrogen bond acceptors (nON), number of rotatable bonds (nRTB) and a polar surface area (PSA) known as Lipinski criteria as well as blood-brain barrier (BBB) penetration (log BB), were estimated and evaluated. All these parameters influence the toxicity and ADME of a drug in human body. There is a great emphasis on the prediction of drug penetration through BBB in order to avoid complications in the later phases of drug development due to undesirable neurotoxicity as a side effect [25]. The penetration through the BBB has been negatively related to molecular properties such as high drug lipophilicity, molecular weight, and the tendency to form hydrogen bonds quantified as 
PSA [26-28]. As shown in Table 3, the discussed compounds do fulfill the Lipinski rules, except the number of rotatable bonds, but the already clinically-used landiolol shows similarities. A large number of rotatable bonds has been associated with poor oral bioavailability, particularly when associated with a high polar surface area [29]. For prediction of the ability of our compounds to cross the BBB by passive transport, we chose the model proposed by Vilar et al. [27]. Based on previously described facts that compounds with $\log \mathrm{BB}>0.3$ pass the $\mathrm{BBB}$ readily and compounds with $\log \mathrm{BB}<-1$ are poorly distributed in the brain [30,31], Vilar et al. generated two distinct classification models for both threshold values. These models were generated using in vivo log BB values of 307 compounds and validated on 1457 molecules using their in silico physicochemical descriptors with the percentage of good classified compounds around $80 \%$ and can be used also for prediction of log BB values of novel compounds. According to the results calculated by model 1 (results shown in Table 3) none of our compounds exceeded the threshold value of 0.3 , suggesting that these compounds could not cross the BBB readily. However the values calculated according to the model 2, which suggests poor penetration through the BBB, are greater than the threshold value $(\log B B>-1)$. Therefore, studied drugs belong to the category of compounds with $\log \mathrm{BB}$ values comprised between 0.3 and -1 , which still have access to the central nervous system (CNS); i.e., the studied compounds can possess low brain permeability. These results should be taken into account during the further stages of drug development.

Table 3. Calculated parameters related to toxicity and absorption, distribution, metabolism, and excretion (ADME).

\begin{tabular}{|c|c|c|c|c|c|c|c|}
\hline Comp. & $\begin{array}{l}\mathrm{MW}^{\mathrm{a}} \\
(<500)\end{array}$ & $\begin{array}{c}\mathrm{nOHNH} \\
(<5)\end{array}$ & $\begin{array}{l}\mathrm{nON} \\
(<10)\end{array}$ & $\begin{array}{l}\text { nRTB } \\
(\leq 10)\end{array}$ & $\begin{array}{c}\text { PSA }_{\text {pH 7.0 }}{ }^{b} \\
\quad(\leq 140)\end{array}$ & $\begin{array}{c}\text { Log BB Class } \\
\text { (Model 1) }\end{array}$ & $\begin{array}{c}\text { Log BB Class } \\
\text { (Model 2) }\end{array}$ \\
\hline $7 a$ & 440.53 & 3 & 7 & 13 & 72.67 & -0.29 & 0.32 \\
\hline $7 \mathbf{b}$ & 470.56 & 3 & 8 & 14 & 81.90 & -0.61 & -0.01 \\
\hline $7 c$ & 470.56 & 3 & 8 & 14 & 81.90 & -0.70 & -0.05 \\
\hline $8 a$ & 442.56 & 3 & 7 & 12 & 72.67 & -0.17 & 0.38 \\
\hline $8 b$ & 472.58 & 3 & 8 & 13 & 81.90 & -0.48 & 0.06 \\
\hline $8 c$ & 472.58 & 3 & 8 & 13 & 81.90 & -0.59 & 0.01 \\
\hline $9 a$ & 442.56 & 3 & 7 & 13 & 72.67 & -0.12 & 0.40 \\
\hline $9 b$ & 472.58 & 3 & 8 & 14 & 81.90 & -0.45 & 0.07 \\
\hline $9 c$ & 472.58 & 3 & 8 & 14 & 81.90 & -0.54 & 0.03 \\
\hline esmolol & 295.37 & 2 & 5 & 10 & 72.37 & $-1.37^{\mathrm{d}}$ & $-0.15^{\mathrm{d}}$ \\
\hline flestolol & 327.35 & 4 & 7 & 9 & 118.26 & $-3.52^{d}$ & $-3.17^{d}$ \\
\hline landiolol & 510.60 & 3 & 11 & 14 & 132.40 & $-3.96^{d}$ & $-3.65^{d}$ \\
\hline
\end{tabular}

${ }^{a}$ Calculated for free bases by ACD ChemSketch; ${ }^{b}$ calculated by MarvinSketch; ${ }^{c}$ calculated from experimental $\left(\log k_{w}\right)$ and calculated (PSA) data according to the models generated by Vilar et al. [27]; ${ }^{\mathrm{d}}$ data for calculation were taken from PubChem. MW, molecular weight; $\mathrm{nOHNH}$, number of hydrogen bond donors; nON, number of hydrogen bond acceptors; nRTB, number of rotatable bonds; PSA, polar surface area; log BB, blood-brain barrier penetration.

\section{Experimental Section}

\subsection{General Information}

All reagents were purchased from Sigma-Aldrich (St. Louis, MO, USA) or Acros Organics (Geel, Belgium) in sufficient purity. The solvents were purchased from Lach-Ner (Neratovice, Czech Republic) and were dried or freshly distilled if necessary. Thin layer chromatography (TLC) Kieselgel $60 \mathrm{~F}_{254}$ plates (Merck, Darmstadt, Germany) visualized by UV irradiation $(254 \mathrm{~nm}$ ) were used to monitor the reactions and the purity of the prepared substances; the reversed-phase TLC RP-18 $\mathrm{F}_{254}$ plates (Merck) were used for the final compounds. The melting points were determined on a Kofler hot-plate apparatus HMK (Franz Kustner Nacht KG, Dresden, Germany) and are uncorrected. The purity of final compounds was analysed by a Dionex Ultimate 3000 (Thermo Fisher Scientific, Waltham, MA, USA) HPLC system controlled through the Chromeleon ${ }^{\circledR}$ Chromatography Data System (version 7.2, Thermo Fisher Scientific). The separation was performed on ZORBAX Extend- $C_{18}$ $(3.5 \mu \mathrm{m}, 3 \times 150 \mathrm{~mm})$ column (Agilent Technologies, Waldbronn, Germany). Mobile phase consisted 
of $0.02 \mathrm{M} 3$-morpholinopropanesulphonate buffer with addition of $0.15 \%$ of $n$-decylamine $(\mathrm{pH}=7.4$; constituent A) and methanol to which $0.25 \%$ of $n$-octanol was added (constituent B). The total flow rate was $0.4 \mathrm{~mL} / \mathrm{min}$, the injection volume was $1 \mu \mathrm{L}$, and the column temperature was maintained at $25{ }^{\circ} \mathrm{C}$. The detection wavelength of $254 \mathrm{~nm}$ was chosen. The purity of individual compounds was calculated as the average of relative peak areas in the chromatograms of the sample solution under isocratic conditions in the range 25:75-50:50 (A:B; v/v). The IR spectra were recorded on a Nicolet Impact 410 FT-IR spectrometer (Thermo Fisher Scientific) using Attenuated Total Reflection (ATR, ZnSe) instrumentation. The spectra were obtained by accumulation of 32 scans in the region 4000-600 $\mathrm{cm}^{-1}$. The instrument was controlled by Omnic v. 8.3 software (Thermo Fisher Scientific). The following abbreviations are used for the characteristic infrared bands: $v(\mathrm{O}-\mathrm{H})$, alcohol $\mathrm{O}-\mathrm{H}$ stretching vibration band; $v(\mathrm{C}-\mathrm{H}), \mathrm{C}-\mathrm{H}$ stretching vibration bands; $v\left(\mathrm{NH}^{+}\right)$, hydrochloride salt of piperazine $\mathrm{N}-\mathrm{H}$ stretching vibration band; $v(\mathrm{C}=\mathrm{O})$, ester $\mathrm{C}=\mathrm{O}$ stretching vibration band; $v($ arom. $\mathrm{C}=\mathrm{C})$, aromatic ring $\mathrm{C}=\mathrm{C}$ stretching vibration band; $v(\mathrm{C}-\mathrm{N})$, piperazine $\mathrm{C}-\mathrm{N}$ stretching vibration band; $v(\mathrm{C}-\mathrm{O}-\mathrm{C})$, ether $\mathrm{C}-\mathrm{O}$ stretching vibration band. The $1 \mathrm{D}\left({ }^{1} \mathrm{H},{ }^{13} \mathrm{C}\left\{{ }^{1} \mathrm{H}\right\},{ }^{13} \mathrm{C}-\mathrm{APT}\right)$ and $2 \mathrm{D}\left({ }^{1} \mathrm{H}-{ }^{1} \mathrm{H} \mathrm{COSY}\right.$, ${ }^{1} \mathrm{H}_{-}{ }^{13} \mathrm{C}$ HMQC and ${ }^{1} \mathrm{H}_{-}{ }^{13} \mathrm{C}$ HMBC) NMR experiments for characterization of the final compounds were performed using a JNM-ECZ4OOR FT-NMR spectrometer $9.39 \mathrm{~T}\left(399.78 \mathrm{MHz}\right.$ for ${ }^{1} \mathrm{H}$ and $100.53 \mathrm{MHz}$ for ${ }^{13} \mathrm{C}$ nucleus; JEOL RESONANCE Inc., Tokyo, Japan) equipped with a $5 \mathrm{~mm}$ High Sensitivity Pulse Field Gradient Autotune ${ }^{\mathrm{TM}}$ probe. Chemical shifts are reported in ppm, referenced to the chemical shifts of residual solvent resonance (DMSO, $2.5 \mathrm{ppm}$ for ${ }^{1} \mathrm{H}$ and $39.5 \mathrm{ppm}$ for ${ }^{13} \mathrm{C}$ ). The coupling constants $(J)$ are reported in Hz. The NMR spectra of intermediates were measured in DMSO- $d_{6}$ on a Gemini-2000 FT-NMR spectrometer (200 MHz for ${ }^{1} \mathrm{H}$ and $50 \mathrm{MHz}$ for ${ }^{13} \mathrm{C}$, Varian Comp., Palo Alto, CA, USA). The following abbreviations are used to describe the NMR signal multiplicities: s, singlet; $d$, doublet; $\mathrm{t}$, triplet; q, quartet; dd, doublet of doublet; dt, doublet of triplet; dq, doublet of quartet; ddt, doublet of doublet of triplet; $m$, multiplet.

High-resolution mass spectra of the final compounds were measured using a high-performance liquid chromatograph Dionex UltiMate ${ }^{\circledR} 3000$ (Thermo Fisher Scientific, West Palm Beach, FL, USA) coupled with a LTQ Orbitrap XL ${ }^{\mathrm{TM}}$ Hybrid Ion Trap-Orbitrap Fourier Transform Mass Spectrometer (Thermo Scientific) with injection into HESI II in the negative mode.

\subsection{Synthesis}

\subsubsection{Synthesis of Tosylate Intermediates}

p-Toluensulfonyl chloride $(2.6 \mathrm{~g}, 0.014 \mathrm{~mol})$ was slowly added to the mixture of appropriate 2-alkoxyethanol respectively (oxiran-2-yl)methanol $(0.014 \mathrm{~mol})$ and triethylamine $(1.84 \mathrm{~g}, 0.018 \mathrm{~mol}) \mathrm{in}$ dichlormethane $(20 \mathrm{~mL})$ cooled at $0^{\circ} \mathrm{C}$. The mixture was then stirred for $1 \mathrm{~h}$ at ambient temperature. The precipitate was filtered and the organic phase was washed with $2 \mathrm{M} \mathrm{HCl}$, saturated solution of $\mathrm{Na}_{2} \mathrm{CO}_{3}$ and water, dried over $\mathrm{MgSO}_{4}$, and then the solvent was evaporated. Product T2 was recrystallized from petroleum ether/diethyl ether in ratio 5:1.

Allyloxyethyl 4-methylbenzensulfonate (T1a). Yield: 97\%; $R_{\mathrm{f}}$ : 0.74 (ethyl acetate/petroleum ether 1:1); ${ }^{1} \mathrm{H}-\mathrm{NMR}\left(200 \mathrm{MHz}, \mathrm{DMSO}-d_{6}\right) \delta(\mathrm{ppm}): 7.79\left(\mathrm{~d}, J=8.5 \mathrm{~Hz}, 2 \mathrm{H}, \mathrm{Ar}-\mathrm{H}^{2,6}\right), 7.48(\mathrm{~d}, J=8.5 \mathrm{~Hz}, 2 \mathrm{H}$, $\left.\mathrm{Ar}-\mathrm{H}^{3,5}\right), 5.79(\mathrm{ddt}, J=17.2,10.4,5.4 \mathrm{~Hz}, 1 \mathrm{H},-\mathrm{CH}=), 5.27-5.07\left(\mathrm{~m}, 2 \mathrm{H},=\mathrm{CH}_{2}\right), 4.15-4.11(\mathrm{~m}, 2 \mathrm{H}$, $\left.\mathrm{TsOCH}_{2}\right), 3.87\left(\mathrm{dt}, J=5.3,1.4 \mathrm{~Hz}, 2 \mathrm{H},-\mathrm{CH}_{2} \mathrm{CH}=\right), 3.56-3.52\left(\mathrm{~m}, 2 \mathrm{H}, \mathrm{CH}_{2} \mathrm{CH}_{2} \mathrm{O}\right), 2.42\left(\mathrm{~s}, 3 \mathrm{H}, \mathrm{ArCH}_{3}\right)$; ${ }^{13} \mathrm{C}-\mathrm{NMR}\left(50 \mathrm{MHz}, \mathrm{DMSO}-d_{6}\right) \delta$ (ppm): 144.77, 134.62, 132.40, 130.00, 127.47, 116.47, 70.78, 69.84, 66.93, 20.98. (CAS Registry Number 50563-72-9).

Isopropoxyethyl 4-methylbenzensulfonate (T1b). Yield: 85\%; $R_{\mathrm{f}}$ : 0.70 (ethyl acetate/petroleum ether 1:1); ${ }^{1} \mathrm{H}-\mathrm{NMR}\left(200 \mathrm{MHz}, \mathrm{DMSO}-\mathrm{d}_{6}\right) \delta(\mathrm{ppm}): 7.78\left(\mathrm{~d}, J=8.5 \mathrm{~Hz}, 2 \mathrm{H}, \mathrm{Ar}-\mathrm{H}^{2,6}\right), 7.48\left(\mathrm{~d}, J=8.5,2 \mathrm{H}, \mathrm{Ar}-\mathrm{H}^{3,5}\right)$, 4.10-4.06 (m, 2H, TsOCH $)_{2}, 3.54-3.41\left(\mathrm{~m}, 3 \mathrm{H}, \mathrm{CH}_{2} \mathrm{CH}_{2} \mathrm{O}+-\mathrm{CH}\left(\mathrm{CH}_{3}\right)_{2}\right), 2.42\left(\mathrm{~s}, 3 \mathrm{H}, \mathrm{ArCH}_{3}\right), 1.00(\mathrm{~d}$, $\left.J=6.0,6 \mathrm{H},-\mathrm{CH}\left(\mathrm{CH}_{3}\right)_{2}\right) ;{ }^{13} \mathrm{C}-\mathrm{NMR}\left(50 \mathrm{MHz}, \mathrm{DMSO}-d_{6}\right) \delta(\mathrm{ppm}): 144.82,132.47,130.06,127.57,70.91$, 70.44, 64.85, 21.76, 21.05. (CAS Registry Number 51218-98-5). 
Propoxyethyl 4-methylbenzensulfonate (T1c). Yield: $86 \% ; R_{\mathrm{f}}$ : 0.72 (ethyl acetate/petroleum ether 1:1); ${ }^{1} \mathrm{H}-\mathrm{NMR}\left(200 \mathrm{MHz}, \mathrm{DMSO}-d_{6}\right) \delta(\mathrm{ppm}): 7.78\left(\mathrm{~d}, J=8.5 \mathrm{~Hz}, 2 \mathrm{H}, \mathrm{Ar}-\mathrm{H}^{2,6}\right), 7.48\left(\mathrm{~d}, J=8.5,2 \mathrm{H}, \mathrm{Ar}-\mathrm{H}^{3,5}\right)$, 4.13-4.09 (m, 2H, TsOCH $), 3.53-3.49\left(\mathrm{~m}, 2 \mathrm{H}, \mathrm{CH}_{2} \mathrm{CH}_{2} \mathrm{O}\right), 3.23\left(\mathrm{t}, J=6.4 \mathrm{~Hz}, 2 \mathrm{H},-\mathrm{CH}_{2} \mathrm{CH}_{2} \mathrm{CH}_{3}\right), 2.42$ $\left(\mathrm{s}, 3 \mathrm{H}, \mathrm{ArCH}_{3}\right), 1.50-1.32\left(\mathrm{~m}, 2 \mathrm{H},-\mathrm{CH}_{2} \mathrm{CH}_{2} \mathrm{CH}_{3}\right), 0.77\left(\mathrm{t}, J=7.7 \mathrm{~Hz}, 3 \mathrm{H},-\mathrm{CH}_{3}\right) ;{ }^{13} \mathrm{C}-\mathrm{NMR}(50 \mathrm{MHz}$, DMSO- $d_{6}$ ) $\delta$ (ppm): 144.85, 132.43, 130.08, 127.57, 71.71, 70.00, 67.39, 22.22, 21.05, 10.32. (CAS Registry Number 52497-47-9).

(Oxiran-2-yl)methyl 4-methylbenzensulfonate (T2). Yield: 97\%; $R_{\mathrm{f}}$ : 0.77 (ethyl acetate/petroleum ether 1:1); m.p.: $35-37^{\circ} \mathrm{C} ;{ }^{1} \mathrm{H}-\mathrm{NMR}\left(200 \mathrm{MHz}\right.$, DMSO- $\left.d_{6}\right) \delta(\mathrm{ppm}): 7.80\left(\mathrm{~d}, J=8.4 \mathrm{~Hz}, 2 \mathrm{H}, \mathrm{Ar}-\mathrm{H}^{2,6}\right), 7.49(\mathrm{~d}$, $\left.J=8.4 \mathrm{~Hz}, 2 \mathrm{H}, \mathrm{Ar}-\mathrm{H}^{3,5}\right), 4.41\left(\mathrm{dd}, J=11.5,2.4 \mathrm{~Hz}, 1 \mathrm{H}, \mathrm{TsOCH}_{2}\right), 3.81\left(\mathrm{dd}, J=11.5,7.1 \mathrm{~Hz}, 1 \mathrm{H}, \mathrm{TsOCH}_{2}\right)$, 3.22-3.14 (m, 1H, CH-oxiran), 2.79-2.74 (m, 1H, $\mathrm{CH}_{2}$-oxiran), 2.60 (dd, J = 5.0, $2.6 \mathrm{~Hz}, 1 \mathrm{H}, \mathrm{CH}_{2}$-oxiran), $2.42\left(\mathrm{~s}, 3 \mathrm{H}, \mathrm{ArCH}_{3}\right) ;{ }^{13} \mathrm{C}-\mathrm{NMR}\left(50 \mathrm{MHz}, \mathrm{DMSO}-d_{6}\right) \delta(\mathrm{ppm}): 144.99,132.20,130.10,127.51,71.58,48.49$, 43.63, 20.98. (CAS Registry Number 118712-54-2).

\subsubsection{Synthesis of 4-Alkoxyethoxybenzoic Acid}

A mixture of ethyl-4-hydroxybenzoate $(3.32 \mathrm{~g}, 0.02 \mathrm{~mol})$, tosylate intermediate T1a-c $(0.02 \mathrm{~mol})$ and potassium carbonate $(0.06 \mathrm{~mol})$ in acetone $(25 \mathrm{~mL})$ was heated at $70{ }^{\circ} \mathrm{C}$ for $8 \mathrm{~h}$ and then stirred for $12 \mathrm{~h}$ at room temperature. The reaction was monitored by means of TLC. The precipitation was then filtered and acetone was evaporated. The residue was dissolved in ethyl acetate and washed with $2 \mathrm{M} \mathrm{NaOH}$ and water, dried over $\mathrm{MgSO}_{4}$, and then the solvent was evaporated. The crude product heated for $2 \mathrm{~h}$ with $5 \mathrm{M} \mathrm{NaOH}(50 \mathrm{~mL})$ at $100{ }^{\circ} \mathrm{C}$. The reaction mixture was washed with chloroform and neutralized with concentrated $\mathrm{HCl}$. The resulting white precipitate was collected by filtration.

4-(2-Allyloxy)ethoxybenzoic acid (3a). Yield: $69 \% ; R_{\mathrm{f}}$ : 0.67 (ethyl acetate); m.p.: $120-123{ }^{\circ} \mathrm{C} ;{ }^{1} \mathrm{H}-\mathrm{NMR}$ $\left(200 \mathrm{MHz}, \mathrm{DMSO}-d_{6}\right) \delta(\mathrm{ppm}): 12.60(\mathrm{bs}, 1 \mathrm{H}, \mathrm{COOH}), 7.88\left(\mathrm{~d}, J=9.0 \mathrm{~Hz}, 2 \mathrm{H}, \mathrm{Ar}-\mathrm{H}^{2,6}\right), 7.02(\mathrm{~d}, J=9.0 \mathrm{~Hz}$, $\left.2 \mathrm{H}, \mathrm{Ar}-\mathrm{H}^{3,5}\right), 5.89(\mathrm{ddt}, J=17.3,10.5,5.3 \mathrm{~Hz}, 1 \mathrm{H},-\mathrm{CH}=), 5.26\left(\mathrm{dq}, J=17.3,1.8 \mathrm{~Hz}, 1 \mathrm{H},=\mathrm{CH}_{2}\right), 5.18-5.11$ $\left(\mathrm{m}, 1 \mathrm{H},=\mathrm{CH}_{2}\right), 4.20-4.16\left(\mathrm{~m}, 2 \mathrm{H}, \mathrm{ArOCH}_{2}\right), 4.01\left(\mathrm{dt}, J=5.3,1.6 \mathrm{~Hz}, 2 \mathrm{H},-\mathrm{CH}_{2} \mathrm{CH}=\right), 3.75-3.71(\mathrm{~m}, 2 \mathrm{H}$, $\left.\mathrm{CH}_{2} \mathrm{O}_{\text {allyl }}\right) ;{ }^{13} \mathrm{C}-\mathrm{NMR}\left(50 \mathrm{MHz}\right.$, DMSO- $d_{6}$ ) $\delta$ (ppm): 166.94, 162.05, 135.03, 131.30, 123.03, 116.52, 114.25, 71.10, 67.91, 67.37. (CAS Registry Number 153881-37-9).

4-(2-Isopropoxy)ethoxybenzoic acid (3b). Yield: $65 \% ; R_{\mathrm{f}}$ : 0.67 (ethyl acetate); m.p.: $128-130{ }^{\circ} \mathrm{C} ;{ }^{1} \mathrm{H}-\mathrm{NMR}$ $\left(200 \mathrm{MHz}, \mathrm{DMSO}-d_{6}\right) \delta(\mathrm{ppm}): 12.61$ (bs, $\left.1 \mathrm{H}, \mathrm{COOH}\right), 7.87\left(\mathrm{~d}, J=9.0 \mathrm{~Hz}, 2 \mathrm{H}, \mathrm{Ar}-\mathrm{H}^{2,6}\right), 7.02(\mathrm{~d}, J=9.0 \mathrm{~Hz}$, $\left.2 \mathrm{H}, \mathrm{Ar}-\mathrm{H}^{3,5}\right), 4.15-4.10\left(\mathrm{~m}, 2 \mathrm{H}, \mathrm{ArOCH}_{2}\right), 3.72-3.56\left(\mathrm{~m}, 3 \mathrm{H},-\mathrm{CH}_{2} \mathrm{OCH}\left(\mathrm{CH}_{3}\right)_{2}\right), 1.10(\mathrm{~d}, J=6.0 \mathrm{~Hz}, 6 \mathrm{H}$, $\left.-\mathrm{CH}\left(\mathrm{CH}_{3}\right)_{2}\right) ;{ }^{13} \mathrm{C}-\mathrm{NMR}\left(50 \mathrm{MHz}\right.$, DMSO- $\left.d_{6}\right) \delta(\mathrm{ppm}): 166.96,162.13,131.30,122.96,114.26,71.07,67.79$, 65.74, 21.99. (CAS Registry Number 766552-53-8).

4-(2-Propoxy)ethoxybenzoic acid (3c). Yield: $69 \% ; R_{\mathrm{f}}: 0.70$ (ethyl acetate); m.p.: $109-111^{\circ} \mathrm{C}$ (lit. $114-115^{\circ} \mathrm{C}$ ); ${ }^{1} \mathrm{H}-\mathrm{NMR}\left(200 \mathrm{MHz}, \mathrm{DMSO}-d_{6}\right) \delta$ (ppm): 12.63 (bs, $\left.1 \mathrm{H}, \mathrm{COOH}\right), 7.85\left(\mathrm{~d}, J=9.0 \mathrm{~Hz}, 2 \mathrm{H}, \mathrm{Ar}-\mathrm{H}^{2,6}\right), 7.02$ $\left(\mathrm{d}, J=9.0 \mathrm{~Hz}, 2 \mathrm{H}, \mathrm{Ar}-\mathrm{H}^{3,5}\right), 4.18-4.13\left(\mathrm{~m}, 2 \mathrm{H}, \mathrm{ArOCH}_{2}\right), 3.72-3.68\left(\mathrm{~m}, 2 \mathrm{H},-\mathrm{CH}_{2} \mathrm{OPr}\right), 3.40(\mathrm{t}, J=6.6 \mathrm{~Hz}$, $\left.2 \mathrm{H},-\mathrm{CH}_{2} \mathrm{CH}_{2} \mathrm{CH}_{3}\right), 1.60-1.43\left(\mathrm{~m}, 2 \mathrm{H},-\mathrm{CH}_{2} \mathrm{CH}_{2} \mathrm{CH}_{3}\right), 0.85\left(\mathrm{t}, \mathrm{J}=7.5 \mathrm{~Hz}, 3 \mathrm{H},-\mathrm{CH}_{2} \mathrm{CH}_{2} \mathrm{CH}_{3}\right) ;{ }^{13} \mathrm{C}-\mathrm{NMR}$ $\left(50 \mathrm{MHz}, \mathrm{DMSO}-d_{6}\right) \delta$ (ppm): 166.96, 162.10, 131.30, 122.99, 114.25, 72.01, 68.35, 67.44, 22.38, 10.44 . (CAS Registry Number: 67132-01-8).

\subsubsection{Synthesis of Oxirane-Intermediates}

A mixture of 4-alkoxyethoxybenzoic acid 3a-c $(0.028 \mathrm{~mol})$ in methanol $(75 \mathrm{~mL})$ and $\mathrm{KOH}(2.2 \mathrm{~g}$, $0.042 \mathrm{~mol})$ in propan-2-ol $(50 \mathrm{~mL})$ was stirred for $1 \mathrm{~h}$ at room temperature, and after that, propan-2-ol (175 mL) was added for the final ratio methanol/propan-2-ol 1:3. The resulting white precipitate was collected by filtration and dried under low pressure. A mixture of appropriate potassium salt ( $5 \mathrm{~g}$, $0.023 \mathrm{~mol}$ ) with (oxiran-2-yl)methyl 4-methylbenzensulfonate T2 (3.5 g, $0.015 \mathrm{~mol})$ in DMF (50 mL) was heated for $7 \mathrm{~h}$ at $70{ }^{\circ} \mathrm{C}$. The reaction was monitored by means of TLC. The solvent was evaporated, and the residue was dissolved in ethyl acetate and washed with water; the organic layer was dried over $\mathrm{MgSO}_{4}$, and ethyl acetate was evaporated. 
(Oxiran-2-yl)methyl 4-allyloxyethoxybenzoate (4). Yield: 88\%; $R_{\mathrm{f}}$ : 0.79 (ethyl acetate/petroleum ether 1:1); ${ }^{1} \mathrm{H}-\mathrm{NMR}\left(200 \mathrm{MHz}\right.$, DMSO- $\left.d_{6}\right) \delta(\mathrm{ppm}): 7.93\left(\mathrm{~d}, J=8.9 \mathrm{~Hz}, 2 \mathrm{H}, \mathrm{Ar}-\mathrm{H}^{2,6}\right), 7.07(\mathrm{~d}, J=8.9 \mathrm{~Hz}, 2 \mathrm{H}$, Ar- $\left.\mathrm{H}^{3,5}\right), 5.90(\mathrm{ddt}, J=17.3,10.5,5.3 \mathrm{~Hz}, 1 \mathrm{H},-\mathrm{CH}=), 5.27\left(\mathrm{dq}, J=17.4,1.8 \mathrm{~Hz}, 1 \mathrm{H},=\mathrm{CH}_{2}\right), 5.15(\mathrm{dq}$, $\left.J=10.5,1.5,1 \mathrm{H},=\mathrm{CH}_{2}\right), 4.60\left(\mathrm{dd}, J=12.4,2.7 \mathrm{~Hz}, 1 \mathrm{H}, \mathrm{COOCH}_{2}\right), 4.21-4.19\left(\mathrm{~m}, 2 \mathrm{H}, \mathrm{ArOCH}_{2}\right), 4.08-4.01$ ( $\left.\mathrm{m}, 3 \mathrm{H}, \mathrm{COOCH}_{2}+-\mathrm{CH}_{2} \mathrm{CH}=\right), 3.75-3.73\left(\mathrm{~m}, 2 \mathrm{H}, \mathrm{CH}_{2} \mathrm{O}_{\text {allyl }}\right), 3.34-3.30(\mathrm{~m}, 1 \mathrm{H}, \mathrm{CH}$-oxirane), 2.84-2.82 (m, $1 \mathrm{H}, \mathrm{CH}_{2}$-oxirane), $2.73-2.71$ (m, $1 \mathrm{H}, \mathrm{CH}_{2}$-oxirane); ${ }^{13} \mathrm{C}-\mathrm{NMR}$ (50 MHz, DMSO- $\left.d_{6}\right) \delta$ (ppm): 165.07, $162.54,135.02,131.34,121.51,116.51,114.52,71.09,67.87,67.46,65.05,49.07,43.83$.

(Oxiran-2-yl)methyl 4-isopropoxyethoxybenzoate (5). Yield: 84\%; $R_{\mathrm{f}}$ : 0.78 (ethyl acetate/petroleum ether 1:1); ${ }^{1} \mathrm{H}-\mathrm{NMR}\left(200 \mathrm{MHz}, \mathrm{DMSO}-d_{6}\right) \delta(\mathrm{ppm}): 7.92\left(\mathrm{~d}, J=8.7 \mathrm{~Hz}, 2 \mathrm{H}, \mathrm{Ar}-\mathrm{H}^{2,6}\right), 7.06(\mathrm{~d}, J=8.7 \mathrm{~Hz}, 2 \mathrm{H}$, $\left.\mathrm{Ar}-\mathrm{H}^{3,5}\right), 4.60\left(\mathrm{dd}, J=12.4,2.5 \mathrm{~Hz}, 1 \mathrm{H}, \mathrm{COOCH}_{2}\right), 4.16-4.14\left(\mathrm{~m}, 2 \mathrm{H}, \mathrm{ArOCH}_{2}-\right), 4.05(\mathrm{dd}, J=12.4,6.4$ $\left.\mathrm{Hz}, 1 \mathrm{H}, \mathrm{COOCH}_{2}\right), 3.72-3.69\left(\mathrm{~m}, 2 \mathrm{H},-\mathrm{CH}_{2} \mathrm{OiPr}\right), 3.67-3.58\left(\mathrm{~m}, 1 \mathrm{H},-\mathrm{CH}\left(\mathrm{CH}_{3}\right)_{2}\right), 3.34-3.30(\mathrm{~m}, 1 \mathrm{H}$, CH-oxirane), 2.84-2.82 (m, 1H, $\mathrm{CH}_{2}$-oxirane), 2.73-2.71 (m, 1H, $\mathrm{CH}_{2}$-oxirane), 1.10 (d, J = $6.0 \mathrm{~Hz}, 6 \mathrm{H}$, $\left.-\mathrm{CH}\left(\mathrm{CH}_{3}\right)_{2}\right) ;{ }^{13} \mathrm{C}-\mathrm{NMR}\left(50 \mathrm{MHz}\right.$, DMSO- $\left.d_{6}\right) \delta$ (ppm): 165.10, 162.62, 131.35, 121.46, 114.55, 71.09, 67.88, $65.71,65.08,49.09,43.84,21.98$.

(Oxiran-2-yl)methyl 4-propoxyethoxybenzoate (6). Yield: 87\%; $R_{\mathrm{f}}$ : 0.69 (ethyl acetate/petroleum ether 1:1); ${ }^{1} \mathrm{H}-\mathrm{NMR}\left(200 \mathrm{MHz}, \mathrm{DMSO}-d_{6}\right) \delta(\mathrm{ppm}): 7.92\left(\mathrm{~d}, J=8.7 \mathrm{~Hz}, 2 \mathrm{H}, \mathrm{Ar}-\mathrm{H}^{2,6}\right), 7.07(\mathrm{~d}, J=8.7 \mathrm{~Hz}, 2 \mathrm{H}$, Ar- $\left.\mathrm{H}^{3,5}\right), 4.60\left(\mathrm{dd}, J=12.4,2.7 \mathrm{~Hz}, 1 \mathrm{H}, \mathrm{COOCH}_{2}\right), 4.19-4.16\left(\mathrm{~m}, 2 \mathrm{H}, \mathrm{ArOCH}_{2}\right), 4.05(\mathrm{dd}, J=12.4,6.4$ $\left.\mathrm{Hz}, 1 \mathrm{H}, \mathrm{COOCH}_{2}\right), 3.72-3.70\left(\mathrm{~m}, 2 \mathrm{H},-\mathrm{CH}_{2} \mathrm{OPr}\right), 3.40\left(\mathrm{t}, J=6.6 \mathrm{~Hz}, 2 \mathrm{H},-\mathrm{CH}_{2} \mathrm{CH}_{2} \mathrm{CH}_{3}\right), 3.34-3.30(\mathrm{~m}$, $1 \mathrm{H}, \mathrm{CH}$-oxirane), 2.84-2.82 (m, $1 \mathrm{H}, \mathrm{CH}_{2}$-oxirane), 2.73-2.71 (m, $1 \mathrm{H}, \mathrm{CH}_{2}$-oxirane), 1.56-1.47 (m, $2 \mathrm{H}$, $\left.-\mathrm{CH}_{2} \mathrm{CH}_{2} \mathrm{CH}_{3}\right), 0.85\left(\mathrm{t}, J=7.3 \mathrm{~Hz}, 3 \mathrm{H},-\mathrm{CH}_{2} \mathrm{CH}_{2} \mathrm{CH}_{3}\right) ;{ }^{13} \mathrm{C}-\mathrm{NMR}\left(50 \mathrm{MHz}, \mathrm{DMSO}-d_{6}\right) \delta(\mathrm{ppm}): 165.10$, $162.60,131.35,121.49,114.53,72.03,68.33,67.54,65.08,49.09,43.84,22.38,10.44$.

\subsubsection{Synthesis of Final Compounds}

Intermediate 4-6 $(0.004 \mathrm{~mol})$ was added to the solution of the corresponding arylpiperazine $(0.004 \mathrm{~mol})$ in propan-2-ol $(15 \mathrm{~mL})$. The reaction mixture was heated at $80^{\circ} \mathrm{C}$ for $1 \mathrm{~h}$ and stirred for $72 \mathrm{~h}$ at room temperature. After that, the reaction mixture was cooled at least for $48 \mathrm{~h}$ at $-18{ }^{\circ} \mathrm{C}$. Precipitating crude base was filtered and dissolved in diethyl ether or chloroform and transformed to their dihydrochloride salt $\mathbf{7 a}-\mathbf{c}, \mathbf{8 a}-\mathbf{c}, \mathbf{9 a}-\mathbf{c}$ by addition of an excess of saturated ethereal hydrogen chloride. The precipitate was filtered and recrystallized from propan-2-ol, if necessary.

1-\{3-[(4-Allyloxyethoxybenzoyl)oxy]-2-hydroxypropyl\}-4-phenylpiperazinediium dichloride (7a). Yield: 23\%; $R_{\mathrm{f}}: 0.87$ (acetone/toluene 3:1); $R_{\mathrm{f}}$ reverse-phase (rev.): 0.51 (0.1 M HCl/acetone 3:2); m.p.: $185-187^{\circ} \mathrm{C}$; HPLC purity (pur.) 91.67\% (254 nm); IR (ATR, $\left.\mathrm{cm}^{-1}\right): v(\mathrm{O}-\mathrm{H})=3289, v(\mathrm{C}-\mathrm{H})=2930, v\left(\mathrm{NH}^{+}\right)=2537$, $v(\mathrm{C}=\mathrm{O})=1697, v($ arom. $\mathrm{C}=\mathrm{C})=1603, v(\mathrm{C}-\mathrm{N})=1248, v(\mathrm{C}-\mathrm{O}-\mathrm{C})=1175 ;{ }^{1} \mathrm{H}-\mathrm{NMR}(399.78 \mathrm{MHz}$, DMSO- $\left.d_{6}\right) \delta(\mathrm{ppm}): 10.75$ (bs, $\left.2 \mathrm{H}, \mathrm{NH}^{+}{ }_{\text {pip }}\right), 8.00\left(\mathrm{~d}, J=8.7 \mathrm{~Hz}, 2 \mathrm{H}, \mathrm{H}_{\mathrm{ArCOO}}\right), 7.28-7.24(\mathrm{~m}, 2 \mathrm{H}$, $\left.\mathrm{H}_{\text {Ar-pip }}\right), 7.07\left(\mathrm{~d}, J=8.7 \mathrm{~Hz}, 2 \mathrm{H}, \mathrm{H}_{\text {ArO }}\right), 7.01-6.97\left(\mathrm{~m}, 2 \mathrm{H}, \mathrm{H}_{\text {Ar-pip }}\right), 6.88-6.84\left(\mathrm{~m}, 1 \mathrm{H}, \mathrm{H}_{\text {Ar-pip }}\right), 5.90$ (ddt, $J=17.4,10.5,5.4 \mathrm{~Hz}, 1 \mathrm{H},-\mathrm{CH}=), 5.27\left(\mathrm{dq}, J=17.4,1.8 \mathrm{~Hz}, 1 \mathrm{H},=\mathrm{CH}_{2}\right), 5.16(\mathrm{dq}, J=10.5,1.8 \mathrm{~Hz}, 1 \mathrm{H}$, $\left.=\mathrm{CH}_{2}\right), 4.52-4.45\left(\mathrm{~m}, 1 \mathrm{H}, \mathrm{CH}_{\mathrm{OH}}\right), 4.23-4.20\left(\mathrm{~m}, 4 \mathrm{H}, \mathrm{COOCH}_{2}+\mathrm{ArOCH}_{2}\right), 4.02(\mathrm{dt}, J=5.4,1.4 \mathrm{~Hz}$, $\left.2 \mathrm{H},-\mathrm{CH}_{2} \mathrm{CH}=\right), 3.84-3.61\left(\mathrm{~m}, 6 \mathrm{H}, \mathrm{H}_{\text {pip }}+\mathrm{CH}_{2} \mathrm{O}_{\text {allyl }}\right), 3.44-3.15\left(\mathrm{~m}, 6 \mathrm{H}, \mathrm{H}_{\text {pip }}+\mathrm{CH}_{2} \mathrm{~N}_{\text {pip }}\right) ;{ }^{13} \mathrm{C}-\mathrm{NMR}$ (100.53 MHz, DMSO- $d_{6}$ ) $\delta$ (ppm): 165.21, 162.57, 149.60, 135.06, 131.62, 129.14, 121.67, 119.93, 116.62, $115.89,114.43,71.13,67.92,67.50,66.18,63.33,58.13,52.13,50.75,45.25,45.14 ;$ HR-MS: $\mathrm{C}_{25} \mathrm{H}_{33} \mathrm{~N}_{2} \mathrm{O}_{5}$ $[\mathrm{M}-\mathrm{H}]^{-}$calculated $441.2384 \mathrm{~m} / \mathrm{z}$, found $441.2383 \mathrm{~m} / \mathrm{z}$.

1-\{3-[(4-Allyloxyethoxybenzoyl)oxy]-2-hydroxypropyl\}-4-(2-methoxyphenyl)piperazinediium dichloride (7b). Yield: $39 \%$; $R_{\mathrm{f}}$ : 0.83 (acetone/toluene 3:1); $R_{\mathrm{f}}$ (rev.): $0.57\left(0.1 \mathrm{M} \mathrm{HCl}\right.$ /acetone 3:2); m.p.: 178-180 ${ }^{\circ} \mathrm{C}$; HPLC pur. 89.14\% (254 nm); IR (ATR, $\left.\mathrm{cm}^{-1}\right): v(\mathrm{O}-\mathrm{H})=3318, v(\mathrm{C}-\mathrm{H})=2967, v\left(\mathrm{NH}^{+}\right)=2351, v(\mathrm{C}=\mathrm{O})$ $=1711, v($ arom. $\mathrm{C}=\mathrm{C})=1603, v(\mathrm{C}-\mathrm{N})=1252, v(\mathrm{C}-\mathrm{O}-\mathrm{C})=1167 ;{ }^{1} \mathrm{H}-\mathrm{NMR}\left(399.78 \mathrm{MHz}, \mathrm{DMSO}-d_{6}\right) \delta$ (ppm): $10.86\left(\mathrm{bs}, 2 \mathrm{H}, \mathrm{NH}^{+}\right.$pip), $8.00\left(\mathrm{~d}, J=8.7 \mathrm{~Hz}, 2 \mathrm{H}, \mathrm{H}_{\text {ArCOO}}\right), 7.07\left(\mathrm{~d}, J=8.7 \mathrm{~Hz}, 2 \mathrm{H}, \mathrm{H}_{\mathrm{ArO}}\right), 7.04-7.02$ (m, 1H, $\left.\mathrm{H}_{\text {Ar-pip }}\right), 7.00-6.97$ (m, 2H, $\left.\mathrm{H}_{\text {Ar-pip }}\right), 6.93-6.89$ (m, 1H, $\left.\mathrm{H}_{\text {Ar-pip }}\right), 5.89$ (ddt, $J=17.4,10.5,5.4 \mathrm{~Hz}$, $1 \mathrm{H},-\mathrm{CH}=), 5.27\left(\mathrm{dq}, J=17.4,1.8 \mathrm{~Hz}, 1 \mathrm{H},=\mathrm{CH}_{2}\right), 5.15\left(\mathrm{dq}, J=10.5,1.8 \mathrm{~Hz}, 1 \mathrm{H},=\mathrm{CH}_{2}\right), 4.52-4.45(\mathrm{~m}$, 
$\left.1 \mathrm{H}, \mathrm{CH}_{\mathrm{OH}}\right), 4.22-4.19\left(\mathrm{~m}, 4 \mathrm{H}, \mathrm{COOCH}_{2}+\mathrm{ArOCH}_{2}\right), 4.01\left(\mathrm{dt}, J=5.4,1.4 \mathrm{~Hz}, 2 \mathrm{H},-\mathrm{CH}_{2} \mathrm{CH}=\right), 3.80$ (s, $\left.3 \mathrm{H}, \mathrm{OCH}_{3}\right), 3.77-3.60\left(\mathrm{~m}, 6 \mathrm{H}, \mathrm{H}_{\text {pip }}+\mathrm{CH}_{2} \mathrm{O}_{\text {allyl }}\right), 3.52-3.13\left(\mathrm{~m}, 6 \mathrm{H}, \mathrm{H}_{\text {pip }}+\mathrm{CH}_{2} \mathrm{~N}_{\text {pip }}\right) ;{ }^{13} \mathrm{C}-\mathrm{NMR}$ $\left(100.53 \mathrm{MHz}, \mathrm{DMSO}-d_{6}\right) \delta$ (ppm): 165.22, 162.57, 151.85, 139.00, 135.08, 131.65, 123.79, 121.70, 120.88, 118.41, 116.62, 114.44, 112.03, 71.14, 67.93, 67.51, 66.18, 63.36, 58.35, 55.44, 52.50, 51.25, 46.90, 46.74; HR-MS: $\mathrm{C}_{26} \mathrm{H}_{35} \mathrm{~N}_{2} \mathrm{O}_{6}[\mathrm{M}-\mathrm{H}]^{-}$calculated $471.2489 \mathrm{~m} / \mathrm{z}$, found $471.2484 \mathrm{~m} / \mathrm{z}$.

1-\{3-[(4-Allyloxyethoxybenzoyl)oxy]-2-hydroxypropyl\}-4-(4-methoxyphenyl)piperazinediium dichloride (7c). Yield: $44 \% ; R_{\mathrm{f}}: 0.82$ (acetone/toluene 3:1); $R_{\mathrm{f}}$ (rev.): $0.58\left(0.1 \mathrm{M} \mathrm{HCl}\right.$ /acetone 3:2); m.p.: $170-173{ }^{\circ} \mathrm{C}$; HPLC pur. $94.28(254 \mathrm{~nm})$; IR $\left(\right.$ ATR, $\left.\mathrm{cm}^{-1}\right): v(\mathrm{O}-\mathrm{H})=3335, v(\mathrm{C}-\mathrm{H})=2978, v\left(\mathrm{NH}^{+}\right)=2377$, $v(\mathrm{C}=\mathrm{O})=1710, v($ arom. $\mathrm{C}=\mathrm{C})=1604, v(\mathrm{C}-\mathrm{N})=1250, v(\mathrm{C}-\mathrm{O}-\mathrm{C})=1166 ;{ }^{1} \mathrm{H}-\mathrm{NMR}(399.78 \mathrm{MHz}$, DMSO-d $\left.d_{6}\right) \delta(\mathrm{ppm}): 10.96\left(\mathrm{bs}, 2 \mathrm{H}, \mathrm{NH}^{+}{ }_{\mathrm{pip}}\right), 8.00\left(\mathrm{~d}, J=8.7 \mathrm{~Hz}, 2 \mathrm{H}, \mathrm{H}_{\mathrm{ArCOO}}\right), 7.10(\mathrm{~d}, J=9.1 \mathrm{~Hz}, 2 \mathrm{H}$, $\left.\mathrm{H}_{\text {Ar-pip }}\right), 7.07\left(\mathrm{~d}, J=8.7 \mathrm{~Hz}, 2 \mathrm{H}, \mathrm{H}_{\text {ArO }}\right), 6.91\left(\mathrm{~d}, 2 \mathrm{H}, J=9.1 \mathrm{~Hz}, \mathrm{H}_{\text {Ar-pip }}\right), 5.89(\mathrm{ddt}, J=17.3,10.5,5.3 \mathrm{~Hz}$, $1 \mathrm{H},-\mathrm{CH}=), 5.27\left(\mathrm{dq}, J=17.3,1.7 \mathrm{~Hz}, 1 \mathrm{H},=\mathrm{CH}_{2}\right), 5.15\left(\mathrm{dq}, J=10.5,1.7 \mathrm{~Hz}, 1 \mathrm{H},=\mathrm{CH}_{2}\right), 4.51-4.46(\mathrm{~m}, 1 \mathrm{H}$, $\left.\mathrm{CH}_{\mathrm{OH}}\right), 4.23-4.19\left(\mathrm{~m}, 4 \mathrm{H}, \mathrm{COOCH}_{2}+\mathrm{ArOCH}_{2}\right), 4.01\left(\mathrm{dt}, J=5.3,1.7 \mathrm{~Hz}, 2 \mathrm{H},-\mathrm{CH}_{2} \mathrm{CH}=\right), 3.79-3.60(\mathrm{~m}$, $\left.6 \mathrm{H}, \mathrm{H}_{\text {pip }}+\mathrm{CH}_{2} \mathrm{O}_{\text {allyl }}\right), 3.71\left(\mathrm{~s}, 3 \mathrm{H}, \mathrm{OCH}_{3}\right), 3.53-3.25\left(\mathrm{~m}, 6 \mathrm{H}, \mathrm{H}_{\text {pip }}+\mathrm{CH}_{2} \mathrm{~N}_{\text {pip }}\right) ;{ }^{13} \mathrm{C}-\mathrm{NMR}(100.53 \mathrm{MHz}$, DMSO- $_{6}$ ) $\delta$ (ppm): 165.23, 162.57, 154.69, 142.13, 135.08, 131.64, 121.69, 118.78, 116.62, 114.56, 114.44, 71.14, 67.93, 67.51, 66.20, 63.46, 58.04, 55.32, 51.82, 50.62, 47.26, 47.19; HR-MS: $\mathrm{C}_{26} \mathrm{H}_{35} \mathrm{~N}_{2} \mathrm{O}_{6}[\mathrm{M}-\mathrm{H}]^{-}$ calculated $471.2489 \mathrm{~m} / \mathrm{z}$, found $471.2485 \mathrm{~m} / \mathrm{z}$.

1-\{2-Hydroxy-3-[(4-isopropoxyethoxybenzoyl)oxy]propyl\}-4-phenylpiperazinediium dichloride (8a). Yield: 22\%; $R_{\mathrm{f}}: 0.85$ (acetone/toluene 3:1); $R_{\mathrm{f}}$ (rev.): 0.25 (0.1 M HCl/acetone 3:2); m.p.: 200-202 ${ }^{\circ} \mathrm{C}$; HPLC pur. $95.12 \%(254 \mathrm{~nm})$; IR $\left(\right.$ ATR, $\left.\mathrm{cm}^{-1}\right): v(\mathrm{O}-\mathrm{H})=3287, v(\mathrm{C}-\mathrm{H})=2972, v\left(\mathrm{NH}^{+}\right)=2559, v(\mathrm{C}=\mathrm{O})=1699$, $v($ arom. $\mathrm{C}=\mathrm{C})=1603, v(\mathrm{C}-\mathrm{N})=1252, v(\mathrm{C}-\mathrm{O}-\mathrm{C})=1180 ;{ }^{1} \mathrm{H}-\mathrm{NMR}\left(399.78 \mathrm{MHz}, \mathrm{DMSO}-d_{6}\right) \delta(\mathrm{ppm})$ : 10.69 (bs, $\left.2 \mathrm{H}, \mathrm{NH}^{+}{ }_{\text {pip }}\right), 7.99\left(\mathrm{~d}, J=8.7 \mathrm{~Hz}, 2 \mathrm{H}, \mathrm{H}_{\text {ArCOO }}\right), 7.28-7.24\left(\mathrm{~m}, 2 \mathrm{H}, \mathrm{H}_{\text {Ar-pip }}\right), 7.06$ (d, J = $8.7 \mathrm{~Hz}$, $\left.2 \mathrm{H}, \mathrm{H}_{\text {ArO }}\right), 7.02-7.00\left(\mathrm{~m}, 2 \mathrm{H}, \mathrm{H}_{\text {Ar-pip }}\right), 6.88-6.84$ (m, 1H, $\left.\mathrm{H}_{\text {Ar-pip }}\right), 4.51-4.44\left(\mathrm{~m}, 1 \mathrm{H}, \mathrm{CH}_{\mathrm{OH}}\right), 4.23-4.21$ $\left(\mathrm{m}, 2 \mathrm{H}, \mathrm{COOCH}_{2}, 4.17-4.14\left(\mathrm{~m}, 2 \mathrm{H}, \mathrm{ArOCH}_{2}\right), 3.84-3.57\left(\mathrm{~m}, 7 \mathrm{H},-\mathrm{CH}_{2} \mathrm{OCH}\left(\mathrm{CH}_{3}\right)_{2}+\mathrm{H}_{\text {pip }}\right), 3.43-3.15\right.$ $\left(\mathrm{m}, 6 \mathrm{H},-\mathrm{CH}_{2} \mathrm{~N}_{\text {pip }}+\mathrm{H}_{\text {pip }}\right), 1.10\left(\mathrm{~d}, J=5.9 \mathrm{~Hz}, 6 \mathrm{H},-\mathrm{CH}\left(\mathrm{CH}_{3}\right)_{2}\right) ;{ }^{13} \mathrm{C}-\mathrm{NMR}\left(100.53 \mathrm{MHz}, \mathrm{DMSO}-d_{6}\right) \delta$ (ppm): 165.23, 162.64, 149.52, 131.62, 129.16, 121.62, 120.03, 115.95, 114.46, 71.13, 67.93, 66.18, 65.76, 63.33, 58.11, 52.14, 50.71, 45.31, 45.19, 22.03; HR-MS: $\mathrm{C}_{25} \mathrm{H}_{36} \mathrm{~N}_{2} \mathrm{O}_{5}[\mathrm{M}-\mathrm{H}]^{-}$calculated $443.2540 \mathrm{~m} / z$, found $443.2535 \mathrm{~m} / \mathrm{z}$.

1-\{2-Hydroxy-3-[(4-isopropoxyethoxybenzoyl)oxy]propyl\}-4-(2-methoxyphenyl)piperazinediium dichloride (8b). Yield: $25 \%$; $R_{\mathrm{f}}$ : 0.82 (acetone/toluene 3:1); $R_{\mathrm{f}}$ (rev.): $0.29\left(0.1 \mathrm{M} \mathrm{HCl} /\right.$ acetone 3:2); m.p.: 154-156 ${ }^{\circ} \mathrm{C}$; HPLC pur. 98.87\% (254 nm); IR (ATR, $\left.\mathrm{cm}^{-1}\right): v(\mathrm{O}-\mathrm{H})=3318, v(\mathrm{C}-\mathrm{H})=2969, v\left(\mathrm{NH}^{+}\right)=2560$, $v(\mathrm{C}=\mathrm{O})=1701, v($ arom. $\mathrm{C}=\mathrm{C})=1603, v(\mathrm{C}-\mathrm{N})=1251, v(\mathrm{C}-\mathrm{O}-\mathrm{C})=1166 ;{ }^{1} \mathrm{H}-\mathrm{NMR}(399.78 \mathrm{MHz}$, DMSO- $\left.d_{6}\right) \delta$ (ppm): 10.67 (bs, $2 \mathrm{H}, \mathrm{NH}^{+}$pip), 7.99 (d, $\left.J=8.7 \mathrm{~Hz}, 2 \mathrm{H}, \mathrm{H}_{\mathrm{ArCOO}}\right), 7.07$ (d, $J=8.7 \mathrm{~Hz}, 2 \mathrm{H}$, $\left.\mathrm{H}_{\text {ArO }}\right), 7.03-6.89\left(\mathrm{~m}, 4 \mathrm{H}, \mathrm{H}_{\text {Ar-pip }}\right), 4.49-4.44\left(\mathrm{~m}, 1 \mathrm{H}, \mathrm{CH}_{\mathrm{OH}}\right), 4.23-4.21\left(\mathrm{~m}, 2 \mathrm{H}, \mathrm{COOCH}_{2}\right), 4.17-4.15(\mathrm{~m}$, $\left.2 \mathrm{H}, \mathrm{ArOCH}_{2}\right), 3.80\left(\mathrm{~s}, 3 \mathrm{H}, \mathrm{OCH}_{3}\right), 3.72-3.70\left(\mathrm{~m}, 2 \mathrm{H},-\mathrm{CH}_{2} \mathrm{O} \mathrm{Pr}\right), 3.68-3.40\left(\mathrm{~m}, 5 \mathrm{H}, \mathrm{H}_{\text {pip }}+-\mathrm{CH}\left(\mathrm{CH}_{3}\right)_{2}\right)$, 3.35-3.08 (m, 6H, $\left.-\mathrm{CH}_{2} \mathrm{~N}_{\text {pip }}+\mathrm{H}_{\text {pip }}\right), 1.11\left(\mathrm{~d}, J=6.4 \mathrm{~Hz}, 6 \mathrm{H},-\mathrm{CH}\left(\mathrm{CH}_{3}\right)_{2}\right) ;{ }^{13} \mathrm{C}-\mathrm{NMR}(100.53 \mathrm{MHz}$, DMSO- $\left.d_{6}\right) \delta$ (ppm): 165.19, 162.62, 151.82, 139.23, 131.60, 123.57, 121.62, 120.84, 118.29, 114.44, 111.96, $71.11,67.91,66.12,65.74,63.27,58.27,55.40,52.63,51.16,46.82,46.67,22.01$; HR-MS: $\mathrm{C}_{26} \mathrm{H}_{37} \mathrm{~N}_{2} \mathrm{O}_{6}$ $[\mathrm{M}-\mathrm{H}]^{-}$calculated $473.2646 \mathrm{~m} / \mathrm{z}$, found $473.2640 \mathrm{~m} / \mathrm{z}$.

1-\{2-Hydroxy-3-[(4-isopropoxyethoxybenzoyl)oxy]propyl\}-4-(4-methoxyphenyl)piperazinediium dichloride (8c). Yield: 34\%; $R_{\mathrm{f}}: 0.81$ (acetone/toluene 3:1); $R_{\mathrm{f}}$ (rev.): $0.23\left(0.1 \mathrm{M} \mathrm{HCl} /\right.$ acetone 3:2); m.p.: 193-195 ${ }^{\circ} \mathrm{C}$; HPLC pur. $95.60(254 \mathrm{~nm})$; IR $\left(\right.$ ATR, $\left.\mathrm{cm}^{-1}\right): v(\mathrm{O}-\mathrm{H})=3270, v(\mathrm{C}-\mathrm{H})=2970, v\left(\mathrm{NH}^{+}\right)=2561$, $v(\mathrm{C}=\mathrm{O})=1698, v($ arom. $\mathrm{C}=\mathrm{C})=1601, v(\mathrm{C}-\mathrm{N})=1247, v(\mathrm{C}-\mathrm{O}-\mathrm{C})=1179 ;{ }^{1} \mathrm{H}-\mathrm{NMR}(399.78 \mathrm{MHz}$, DMSO- $\left.d_{6}\right) \delta$ (ppm): 10.74 (bs, $\left.2 \mathrm{H}, \mathrm{NH}^{+}{ }_{\text {pip }}\right), 7.99\left(\mathrm{~d}, J=8.7 \mathrm{~Hz}, 2 \mathrm{H}, \mathrm{H}_{\mathrm{ArCOO}}\right), 7.07-7.03(\mathrm{~m}, 4 \mathrm{H}$, $\left.\mathrm{H}_{\mathrm{ArO}}+\mathrm{H}_{\text {Ar-pip }}\right), 6.89\left(\mathrm{~d}, J=9.1 \mathrm{~Hz}, 2 \mathrm{H}, \mathrm{H}_{\text {Ar-pip }}\right), 4.50-4.44\left(\mathrm{~m}, 1 \mathrm{H}, \mathrm{CH}_{\mathrm{OH}}\right), 4.23-4.21\left(\mathrm{~m}, 2 \mathrm{H}, \mathrm{COOCH}_{2}\right)$, 4.17-4.14 (m, 2H, $\mathrm{ArOCH}$ ), 3.76-3.57 (m, 10H, $\left.-\mathrm{CH}_{2} \mathrm{OCH}\left(\mathrm{CH}_{3}\right)_{2}+\mathrm{OCH}_{3}+\mathrm{H}_{\text {pip }}\right), 3.45-3.22(\mathrm{~m}, 6 \mathrm{H}$, $\left.-\mathrm{CH}_{2} \mathrm{~N}_{\text {pip }}+\mathrm{H}_{\text {pip }}\right), 1.10\left(\mathrm{~d}, J=5.9 \mathrm{~Hz}, 6 \mathrm{H},-\mathrm{CH}\left(\mathrm{CH}_{3}\right)_{2}\right) ;{ }^{13} \mathrm{C}-\mathrm{NMR}\left(100.53 \mathrm{MHz}, \mathrm{DMSO}-d_{6}\right) \delta(\mathrm{ppm})$ : 165.24, 162.63, 154.24, 142.82, 131.62, 121.63, 118.41, 114.48, 114.46, 71.13, 67.93, 66.17, 65.76, 63.38, 58.05, 
55.28, 52.06, 50.69, 46.96, 46.87, 22.03; HR-MS: $\mathrm{C}_{26} \mathrm{H}_{37} \mathrm{~N}_{2} \mathrm{O}_{6}[\mathrm{M}-\mathrm{H}]^{-}$calculated $473.2646 \mathrm{~m} / z$, found $473.2641 \mathrm{~m} / \mathrm{z}$.

1-\{2-Hydroxy-3-[(4-propoxyethoxybenzoyl)oxy]propyl\}-4-phenylpiperazinediium dichloride (9a). Yield: 32\%; $R_{\mathrm{f}}: 0.85$ (acetone/toluene 3:1); $R_{\mathrm{f}}$ (rev.): $0.20\left(0.1 \mathrm{M} \mathrm{HCl} /\right.$ acetone 3:2); m.p.: 197-199 ${ }^{\circ} \mathrm{C}$; HPLC pur. $96.27 \%(254 \mathrm{~nm}) ; \mathrm{IR}\left(\mathrm{ATR}, \mathrm{cm}^{-1}\right): v(\mathrm{O}-\mathrm{H})=3295, v(\mathrm{C}-\mathrm{H})=2958, v\left(\mathrm{NH}^{+}\right)=2351, v(\mathrm{C}=\mathrm{O})=1702$, $v($ arom. $\mathrm{C}=\mathrm{C})=1601, v(\mathrm{C}-\mathrm{N})=1250, v(\mathrm{C}-\mathrm{O}-\mathrm{C})=1161 ;{ }^{1} \mathrm{H}-\mathrm{NMR}\left(399.78 \mathrm{MHz}, \mathrm{DMSO}-d_{6}\right) \delta(\mathrm{ppm})$ : 10.88 (bs, $2 \mathrm{H}, \mathrm{NH}^{+}$pip), 7.99 (d, J = 8.7 Hz, 2H, $\left.\mathrm{H}_{\text {ArCOO }}\right), 7.28-7.24\left(\mathrm{~m}, 2 \mathrm{H}, \mathrm{H}_{\text {Ar-pip }}\right), 7.06(\mathrm{~d}, J=8.7 \mathrm{~Hz}$, 2H, $\left.\mathrm{H}_{\mathrm{ArO}}\right), 7.03-7.01$ (m, 2H, $\left.\mathrm{H}_{\text {Ar-pip }}\right), 6.89-6.85$ (m, 1H, $\left.\mathrm{H}_{\text {Ar-pip }}\right), 4.52-4.46\left(\mathrm{~m}, 1 \mathrm{H}, \mathrm{CH}_{\mathrm{OH}}\right), 4.23-4.21$ $\left(\mathrm{m}, 2 \mathrm{H}, \mathrm{COOCH}_{2}\right), 4.19-4.17\left(\mathrm{~m}, 2 \mathrm{H}, \mathrm{ArOCH}_{2}\right), 3.83-3.61\left(\mathrm{~m}, 6 \mathrm{H},-\mathrm{CH}_{2} \mathrm{OPr}+\mathrm{H}_{\text {pip }}\right), 3.44-3.15(\mathrm{~m}$, $\left.8 \mathrm{H},-\mathrm{CH}_{2} \mathrm{CH}_{2} \mathrm{CH}_{3}+\mathrm{CH}_{2} \mathrm{~N}_{\text {pip }}+\mathrm{H}_{\text {pip }}\right), 1.55-1.47\left(\mathrm{~m}, 2 \mathrm{H},-\mathrm{CH}_{2} \mathrm{CH}_{2} \mathrm{CH}_{3}\right), 0.85(\mathrm{t}, J=7.5 \mathrm{~Hz}, 3 \mathrm{H}$, $\left.-\mathrm{CH}_{2} \mathrm{CH}_{2} \mathrm{CH}_{3}\right) ;{ }^{13} \mathrm{C}-\mathrm{NMR}\left(100.53 \mathrm{MHz}\right.$, DMSO- $\left.d_{6}\right) \delta$ (ppm): 165.23, 162.59, 149.42, 131.62, 129.17, $121.66,120.17,116.03,114.44,72.04,68.35,67.58,66.21,63.39,58.16,52.03,50.79,45.38,45.26,22.40$, 10.50; HR-MS: $\mathrm{C}_{23} \mathrm{H}_{35} \mathrm{~N}_{2} \mathrm{O}_{5}[\mathrm{M}-\mathrm{H}]^{-}$calculated $443.2540 \mathrm{~m} / \mathrm{z}$, found $443.2534 \mathrm{~m} / z$.

1-\{2-Hydroxy-3-[(4-propoxyethoxybenzoyl)oxy]propyl\}-4-(2-methoxyphenyl)piperazinediium dichloride (9b). Yield: $28 \%$; $R_{\mathrm{f}}$ : 0.81 (acetone/toluene 3:1); $R_{\mathrm{f}}$ (rev.): $0.24\left(0.1 \mathrm{M} \mathrm{HCl}\right.$ /acetone 3:2); m.p.: $143-146{ }^{\circ} \mathrm{C}$; HPLC pur. 98.29\% $(254 \mathrm{~nm})$; IR $\left(\right.$ ATR, $\left.\mathrm{cm}^{-1}\right): v(\mathrm{O}-\mathrm{H})=3290, v(\mathrm{C}-\mathrm{H})=2966, v\left(\mathrm{NH}^{+}\right)=2350$, $v(\mathrm{C}=\mathrm{O})=1700, v($ arom. $\mathrm{C}=\mathrm{C})=1605, v(\mathrm{C}-\mathrm{N})=1247, v(\mathrm{C}-\mathrm{O}-\mathrm{C})=1170 ;{ }^{1} \mathrm{H}-\mathrm{NMR}(399.78 \mathrm{MHz}$, DMSO- $\left.d_{6}\right) \delta$ (ppm): 10.70 (bs, $2 \mathrm{H}, \mathrm{NH}^{+}$pip), $7.99\left(\mathrm{~d}, J=8.7 \mathrm{~Hz}, 2 \mathrm{H}, \mathrm{H}_{\mathrm{ArCOO}}\right), 7.07(\mathrm{~d}, J=8.7 \mathrm{~Hz}, 2 \mathrm{H}$, $\left.\mathrm{H}_{\mathrm{ArO}}\right), 7.03-6.89\left(\mathrm{~m}, 4 \mathrm{H}, \mathrm{H}_{\text {Ar-pip }}\right), 4.50-4.44\left(\mathrm{~m}, 1 \mathrm{H}, \mathrm{CH}_{\mathrm{OH}}\right), 4.23-4.21\left(\mathrm{~m}, 2 \mathrm{H}, \mathrm{COOCH}_{2}\right), 4.20-4.18$ $\left(\mathrm{m}, 2 \mathrm{H}, \mathrm{ArOCH}_{2}-\right), 3.80\left(\mathrm{~s}, 3 \mathrm{H}, \mathrm{OCH}_{3}\right), 3.73-3.70\left(\mathrm{~m}, 2 \mathrm{H},-\mathrm{CH}_{2} \mathrm{OPr}\right), 3.69-3.46\left(\mathrm{~m}, 4 \mathrm{H}, \mathrm{H}_{\text {pip }}\right), 3.41$ $\left(\mathrm{t}, J=6.6 \mathrm{~Hz}, 2 \mathrm{H},-\mathrm{CH}_{2} \mathrm{CH}_{2} \mathrm{CH}_{3}\right), 3.32-3.09\left(\mathrm{~m}, 6 \mathrm{H},-\mathrm{CH}_{2} \mathrm{~N}_{\text {pip }}+\mathrm{H}_{\text {pip }}\right), 1.56-1.47\left(\mathrm{~m}, 2 \mathrm{H},-\mathrm{CH}_{2} \mathrm{CH}_{2} \mathrm{CH}_{3}\right)$, $0.86\left(\mathrm{t}, J=7.3 \mathrm{~Hz}, 3 \mathrm{H},-\mathrm{CH}_{2} \mathrm{CH}_{2} \mathrm{CH}_{3}\right) ;{ }^{13} \mathrm{C}-\mathrm{NMR}\left(100.53 \mathrm{MHz}, \mathrm{DMSO}-d_{6}\right) \delta$ (ppm): 165.19, 162.59, $151.82,139.21,131.61,123.60,121.65,120.85,118.30,114.43,111.98,72.03,68.34,67.57,66.13,63.29,58.29$, 55.40, 52.61, 51.20, 46.83, 46.69, 22.38, 10.47; HR-MS: $\mathrm{C}_{26} \mathrm{H}_{37} \mathrm{~N}_{2} \mathrm{O}_{6}[\mathrm{M}-\mathrm{H}]^{-}$calculated $473.2646 \mathrm{~m} / \mathrm{z}$, found $473.2642 \mathrm{~m} / \mathrm{z}$.

1-\{2-Hydroxy-3-[(4-propoxyethoxybenzoyl)oxy]propyl\}-4-(4-methoxyphenyl)piperazinediium dichloride (9c). Yield: $25 \% ; R_{\mathrm{f}}: 0.81$ (acetone/toluene 3:1); $R_{\mathrm{f}}$ (rev.): 0.22 (0.1 M HCl/acetone 3:2); m.p.: 173-175 ${ }^{\circ} \mathrm{C}$; HPLC pur. $95.52(254 \mathrm{~nm})$; IR $\left(\right.$ ATR, $\left.\mathrm{cm}^{-1}\right): v(\mathrm{O}-\mathrm{H})=3279, v(\mathrm{C}-\mathrm{H})=2934, v\left(\mathrm{NH}^{+}\right)=2562$, $v(\mathrm{C}=\mathrm{O})=1700, v($ arom. $\mathrm{C}=\mathrm{C})=1601, v(\mathrm{C}-\mathrm{N})=1246, v(\mathrm{C}-\mathrm{O}-\mathrm{C})=1182 ;{ }^{1} \mathrm{H}-\mathrm{NMR}(399.78 \mathrm{MHz}$, DMSO- $\left.d_{6}\right) \delta(\mathrm{ppm}): 10.90$ (bs, $\left.2 \mathrm{H}, \mathrm{NH}^{+}{ }_{\mathrm{pip}}\right), 7.99\left(\mathrm{~d}, J=8.7 \mathrm{~Hz}, 2 \mathrm{H}, \mathrm{H}_{\mathrm{ArCOO}}\right), 7.08-7.05(\mathrm{~m}, 4 \mathrm{H}$, $\left.\mathrm{H}_{\mathrm{ArO}}+\mathrm{H}_{\text {Ar-pip }}\right), 6.89\left(\mathrm{~d}, J=9.1 \mathrm{~Hz}, 2 \mathrm{H}, \mathrm{H}_{\text {Ar-pip }}\right), 4.52-4.46\left(\mathrm{~m}, 1 \mathrm{H}, \mathrm{CH}_{\mathrm{OH}}\right), 4.23-4.21(\mathrm{~m}, 2 \mathrm{H}$, $\left.\mathrm{COOCH}_{2}\right), 4.19-4.17\left(\mathrm{~m}, 2 \mathrm{H}, \mathrm{ArOCH}_{2}\right), 3.78-3.63\left(\mathrm{~m}, 9 \mathrm{H},-\mathrm{CH}_{2} \mathrm{OPr}+\mathrm{OCH}_{3}+\mathrm{H}_{\text {pip }}\right), 3.45-3.22(\mathrm{~m}$, $\left.8 \mathrm{H},-\mathrm{CH}_{2} \mathrm{CH}_{2} \mathrm{CH}_{3}+-\mathrm{CH}_{2} \mathrm{~N}_{\text {pip }}+\mathrm{H}_{\text {pip }}\right), 1.56-1.47\left(\mathrm{~m}, 2 \mathrm{H},-\mathrm{CH}_{2} \mathrm{CH}_{2} \mathrm{CH}_{3}\right), 0.85(\mathrm{t}, J=7.5 \mathrm{~Hz}, 3 \mathrm{H}$, $\left.-\mathrm{CH}_{2} \mathrm{CH}_{2} \mathrm{CH}_{3}\right) ;{ }^{13} \mathrm{C}-\mathrm{NMR}\left(100.53 \mathrm{MHz}\right.$, DMSO- $\left.d_{6}\right) \delta$ (ppm): 165.22, 162.60, 154.49, 142.41, 131.63, 121.65, 118.61, 114.52, 114.44, 72.04, 68.35, 67.57, 66.19, 63.43, 58.05, 55.29, 51.89, 50.65, 47.13, 47.05, 22.40, 10.50; HR-MS: $\mathrm{C}_{26} \mathrm{H}_{37} \mathrm{~N}_{2} \mathrm{O}_{6}[\mathrm{M}-\mathrm{H}]^{-}$calculated $473.2646 \mathrm{~m} / \mathrm{z}$, found $473.2643 \mathrm{~m} / \mathrm{z}$.

\subsection{Determination of Physicochemical Parameters}

The $\log k_{w}{ }^{7.4}$ values of synthetized compounds were measured by a Dionex Ultimate 3000 (Thermo Fisher Scientific) HPLC system controlled through the Chromeleon ${ }^{\circledR}$ Chromatography Data System (version 7.2). The separation was performed on ZORBAX Extend- $C_{18}(3.5 \mu \mathrm{m}, 3 \times 150 \mathrm{~mm})$ column (Agilent Technologies). Mobile phase consists of $0.02 \mathrm{M} 3$-morphol, that can be used with addition of $0.15 \%$ of $n$-decylamine ( $\mathrm{pH}=7.4$; constituent $\mathrm{A}$ ) and methanol to which $0.25 \%$ of $n$-octanol was added (constituent B). The total flow rate was $0.4 \mathrm{~mL} / \mathrm{min}$, the injection volume was $1 \mu \mathrm{L}$, and the column temperature was maintained at $25^{\circ} \mathrm{C}$. The detection wavelength of $254 \mathrm{~nm}$ was chosen. One milligram of the sample was dissolved in $1 \mathrm{~mL}$ of methanol and diluted to the concentration $0.1 \mathrm{mg} / \mathrm{mL}$ with $50 \%$ methanol. Retention factors of the compound were measured under isocratic conditions in the range 20:80-50:50 (A:B; $v / v), 5$ values for each factor in duplicate. Linear regression analysis was performed and $\log k_{w}^{7.4}$ values were calculated according to Equation (1). 


$$
\log k=-S \phi+\log k_{w}
$$

where $\log k$ represents a logarithm of individual isocratic factor, $\phi$ is the organic phase concentration, and $S$ is a constant derived by linear regression analysis. The retention factor corresponding to the neutral form $\left(\log k_{w}\right)$ was estimated from the apparent $\log k_{w}^{7.4}$ by using Equation (2).

$$
\log k_{w}=\log k_{w}^{a p p}+\log \left(1+10^{p K a-p H}\right)
$$

The CE experiments were carried out with an Agilent 3D CE (Agilent Technologies) capillary electrophoresis system equipped with an autosampler, automatic injector, photodiode array detector, and an air cooling unit for the capillary. The instrument control and analysis were performed with 3D-CE ChemStation software (Agilent Technologies). Uncoated fused-silica capillary (Agilent Technologies) of $50 \mu \mathrm{m}$ internal diameter, total length of $33.0 \mathrm{~cm}$ and effective length (to the detector) of $24.5 \mathrm{~cm}$ was used. The capillary cassette temperature was maintained at $25{ }^{\circ} \mathrm{C}$ with air cooling. Three milligrams of the sample were dissolved in $1 \mathrm{~mL}$ of $50 \%$ methanol and 10 times diluted with appropriate background electrolyte before analysis. Samples were injected hydrodynamically at 40 mbar pressure for $4.0 \mathrm{~s}$. Running voltage was $15 \mathrm{kV}$ of positive polarity. UV detection was performed at $254 \mathrm{~nm}$. As a marker of electroosmotic flow, mesityl oxide was used. The effective mobilities were recorded for BGE pH values in the range of 3.9 to 9.2 (ionic strength equal to $10 \mathrm{mM}$ ), 4 runs at 10 different buffer $\mathrm{pH}$ values. The effective mobilities $\left(\mu_{\text {eff }}\right)$ were calculated according to Equation (3).

$$
\mu_{e f f}=\frac{L_{t o t} L_{e f f}}{U}\left(\frac{1}{t_{m i g}}-\frac{1}{t_{E O F}}\right)
$$

where $t_{m i g}$ is the migration time of the analyte and $t_{E O F}$ is the migration time of a neutral marker compound that is of the same velocity as the electroosmotic flow (EOF). $L_{\text {eff }}$ is distance from the injection end of capillary to the detector and $L_{t o t}$ is the total length of the capillary over which the voltage $U$ was applied. SigmaPlot for Windows version 11.0 (Systat Software GmbH, Erkrath, Germany) was employed for the nonlinear regression analysis of $\mu_{\text {eff }} / \mathrm{pH}$ relationship having characteristic sigmoidal shape with inflection point indicating $\mathrm{p} K_{\mathrm{a}}$ value.

The $\mathrm{pH}$ measurements were taken with a combination glass electrode (HI 1332B, HANNA Instruments, Woonsocket, RI, USA), using a Thermo Orion 370 PerpHect ${ }^{\circledR} \mathrm{pH}$ meter (ThermoFisher Scientific).

\section{Conclusions}

In conclusion, nine new potential dual $\beta$ - and $\alpha_{1}$-blockers were prepared. In addition, three oxiran intermediates (compounds 4-6) have not yet been described in the literature. In this study, the lipophilicity indexes and the $\mathrm{p} K_{\mathrm{a}}$ values have been experimentally determined and compared with software calculated values. Furthermore, we discussed the ADME properties and potential toxicity of prepared compounds by computing the parameters involved in the Lipinski Rule of Five and the log $\mathrm{BB}$ parameter describing the possibility of the compound to penetrate through the BBB.

Acknowledgments: This study was supported by IGA VFU Brno 323/2016/FaF, 318/2016/FaF. The HPLC/HRMS system forms a part of the National Infrastructure CzeCOS (LM2015061); Michal Oravec was supported by the National Sustainability Program (NPU I; Grant No. LO1415).

Author Contributions: Pavlina Marvanova, Ondrej Hosik, Tereza Padrtova, Petr Mokry—synthesis and structural characterization of the compounds, writing of the paper. Michal Oravec-LC-HRMS analysis of the compounds. Klara Odehnalova-determination of physicochemical parameters, writing of the paper.

Conflicts of Interest: The authors declare no conflict of interest. 


\section{References}

1. Pedersen, M.E.; Cockcroft, J.R. The vasodilatory Beta-blockers. Curr. Hypertens. Rep. 2007, 9, $269-277$. [CrossRef] [PubMed]

2. Reiter, M.-J. Cardiovascular Drug Class Specifity: $\beta$-blockers. Prog. Cardiovasc. Dis. 2004, 47, 11-33. [CrossRef] [PubMed]

3. Chiu, C.C.; Wu, J.R.; Lee, C.H.; Liou, S.F.; Dai, Z.K.; Chen, I.J.; Yeh, J.L. Anti-Hypertension Effect of Vanylidilol: A Phenylaldehyde $\alpha / \beta$-Adrenoceptor Blocker with Endothelium-Dependent and $\mathrm{K}^{+}$Channels Opening-Associated Vasorelaxant Activities. Pharmacology 2004, 70, 140-151. [CrossRef] [PubMed]

4. Deák, K.; Takács-Novák, K.; Kapás, M.; Vastag, M.; Tihanyi, K.; Noszál, B. Physico-chemical characterization of a novel group of dopamine $\mathrm{D}_{3} / \mathrm{D}_{2}$ receptor ligands, potential atypical antipsychotic agents. J. Pharm. Biomed. Anal. 2008, 48, 678-684. [CrossRef] [PubMed]

5. Tengler, J.; Kapustikova, I.; Stropnicky, O.; Mokry, P.; Oravec, M.; Csollei, J.; Jampilek, J. Synthesis of new (arylcarbonyloxy)aminopropanol derivatives and the determination of their physico-chemical properties. Cent. Eur. J. Chem. 2013, 11, 1757-1767. [CrossRef]

6. Mälkiä, A.; Murtomäki, L.; Urtti, A.; Kontturi, K. Drug permeation in biomembranes in vitro and in silico prediction and influence of physicochemical properties. Eur. J. Pharm. Sci. 2004, 23, 13-47. [CrossRef] [PubMed]

7. Alavijeh, M.S.; Chishty, M.; Qaiser, M.Z.; Palmer, A.M. Drug metabolism and pharmacokinetics, the blood-brain barrier, and central nervous system drug discovery. NeuroRx 2005, 2, 554-571. [CrossRef] [PubMed]

8. Duchowicz, P.R.; Castro, E.A. QSPR studies on aqueous solubilities of drug-like compounds. Int. J. Mol. Sci. 2009, 10, 2558-2577. [CrossRef] [PubMed]

9. Mannhold, A.; Waterbeemd, H. Substructure and whole molecule approaches for calculating $\log$ P. J. Comput. Aided Mol. Des. 2001, 15, 337-354. [CrossRef] [PubMed]

10. Giaginis, C.; Tsantili-Kakoulidou, A. Current state of the art in HPLC methodology for lipophilicity assessment of basic drugs. A review. J. Liq. Chromatogr. Relat. Technol. 2008, 31, 79-96. [CrossRef]

11. Lombardo, F.; Shalaeva, M.Y.; Tupper, K.A.; Gao, F. ElogD oct: A tool for lipophilicity determination in drug discovery. 2. Basic and neutral compounds. J. Med. Chem. 2001, 44, 2490-2497. [CrossRef] [PubMed]

12. Stella, C.; Galland, A.; Xiangli, L.; Testa, B.; Rudaz, S.; Veuthey, J.L.; Carrupt, P.A. Novel RPLC stationary phases for lipophilicity measurement: Solvatochromic analysis of retention mechanisms for neutral and basic compounds. J. Sep. Sci. 2004, 27, 284-292. [CrossRef] [PubMed]

13. Reijenga, J.; van Hoof, A.; van Loon, A.; Teunissen, B. Development of methods for the determination of $\mathrm{p} K_{\mathrm{a}}$ Values. Anal. Chem. Insights 2013, 8, 53-71. [CrossRef] [PubMed]

14. Kapustikova, I.; Tengler, J. Determination of Acid-Base Dissociation Constant of Selected $\beta$-Blockers Using Capillary Zone Electrophoresis, RP-HPLC and ${ }^{1} \mathrm{H}-\mathrm{NMR}$. Chem. Listy 2014, 108, 1073-1079.

15. Poole, S.K.; Patel, S.; Dehring, K.; Workman, H.; Poole, C.F. Determination of acid dissociation constants by capillary electrophoresis. J. Chromatogr. A 2004, 1037, 445-454. [CrossRef] [PubMed]

16. Nowak, P.; Woźniakiewicz, M.; Kościelniak, P. Application of capillary electrophoresis in determination of acid dissociation constant values. J. Chromatogr. A 2015, 1377, 1-12. [CrossRef] [PubMed]

17. Andrasi, M.; Buglyo, P.; Zekany, L.; Gaspar, A. A comparative study of capillary zone electrophoresis and pH-potentiometry for determination of dissociation constants. J. Pharm. Biomed. Anal. 2007, 44, 1040-1047. [CrossRef] [PubMed]

18. Bodor, N.; Buchwald, P. Soft drug design: General principles and recent applications. Med. Res. Rev. 2000, 20, 58-101. [CrossRef]

19. Handzlik, J.; Pertz, H.H.; Görnemann, T.; Jähnichen, S.; Kieć-Kononowicz, K. Search for influence of spatial properties on affinity at $\alpha_{1}$-adrenoreceptor subtypes for phenylpiperazine derivatives of phenytoin. Bioorg. Med. Chem. Lett. 2010, 20, 6152-6156. [CrossRef] [PubMed]

20. Wang, Z.; Pei, Y.; Zhao, J.; Li, Z.; Chen, Y.; Zhuo, K. Formation of Ether-Functionalized Ionic-Liquid-Based Aqueous Two-Phase Systems and Their Application in Separation of Protein and Saccharides. J. Phys. Chem. B 2015, 119, 4471-4478. [CrossRef] [PubMed] 
21. Colley, H.E.; Muthana, M.; Danson, S.J.; Jackson, L.V.; Brett, M.L.; Harrison, J.; Coole, S.F.; Mason, D.P.; Jennings, L.R.; Wong, M.; et al. An Orally Bioavailable, Indole-3-glyoxylamide Based Series of Tubulin Polymerization Inhibitors Showing Tumor Growth Inhibition in a Mouse Xenograft Model of Head and Neck Cancer. J. Med. Chem. 2015, 58, 9309-9333. [CrossRef] [PubMed]

22. Ayme, J.-F.; Lux, J.; Sauvage, J.-P.; Sour, A. Catenanes Built Around Octahedral Transition-Metal Complexes that Contain Two Intertwined Endocyclic but Non-sterically Hindering Tridentate Ligands. Chem. Eur. J. 2012, 18, 5565-5573. [CrossRef] [PubMed]

23. Ammazzalorso, A.; Amoroso, R.; Bettoni, G.; Fantacuzzi, M.; De Filippis, B.; Giampietro, L.; Maccallini, C.; Paludi, D.; Tricca, M.L. Synthesis and antibacterial evaluation of oxazolidin-2-ones structurally related to linezolid. Farmaco 2004, 59, 685-690. [CrossRef] [PubMed]

24. Marvanova, P.; Padrtova, T.; Pekarek, T.; Brus, J.; Czernek, J.; Mokry, P.; Humpa, O.; Oravec, M.; Jampilek, J. Synthesis and Characterization of New 3-(4-Arylpiperazin-1-yl)-2-hydroxypropyl 4-Propoxybenzoates and Their Hydrochloride Salts. Molecules 2016, 21, 707. [CrossRef] [PubMed]

25. McAinsh, J.; Cruickshank, J.M. Beta-blockers and central nervous system side effects. Pharmacol. Ther. 1990, 46, 163-197. [CrossRef]

26. Clark, D.E. In silico prediction of blood-brain barrier permeation. Drug Discov. Today 2003, 8, 927-933. [CrossRef]

27. Vilar, S.; Chakrabarti, M.; Costanzi, S. Prediciton of passive blood-brain partitioning: Straightforward and effective classification models based on in silico derived physicochemical descriptors. J. Mol. Graph. Model. 2010, 28, 899-903. [CrossRef] [PubMed]

28. Palmer, A.M.; Alavijeh, M.S. Translation CNS medicines research. Drug Discov. Today 2012, 17, $1068-1078$. [CrossRef] [PubMed]

29. Veber, D.F.; Johnson, S.R.; Cheng, H.Y.; Smith, B.R.; Ward, K.W.; Kopple, K.D. Molecular properties that influence the oral bioavailability of drug candidate. J. Med. Chem. 2002, 45, 2615-2623. [CrossRef] [PubMed]

30. Clark, D.E. Rapid calculation of polar molecular surface area and its application to the prediction of transport phenomena. 2. Prediction of blood-brain barrier penetration. J. Pharm. Sci. 1999, 88, 815-821. [CrossRef] [PubMed]

31. Hutter, M.C. Prediction of blood-brain barrier permeation using quantum chemically derived information. J. Comput. Aided Mol. Des. 2003, 17, 415-433. [CrossRef] [PubMed]

Sample Availability: Samples of compounds are available from authors P. Marvanova, O. Hosik and P. Mokry.

(C) 2016 by the authors; licensee MDPI, Basel, Switzerland. This article is an open access article distributed under the terms and conditions of the Creative Commons Attribution (CC-BY) license (http:/ / creativecommons.org/licenses/by/4.0/). 